\title{
User's Guide for MapMark4GUI-A Graphical User Interface for the MapMark4 R Package
}

Chapter 18 of

Section C, Computer Programs

Book 7, Automated Data Processing and Computations

Software support for probability calculations in three-part mineral resource assessments

Techniques and Methods 7-C18 



\title{
User's Guide for MapMark4GUI_A Graphical User Interface for the MapMark4 R Package
}

\author{
By Jason Shapiro
}

Chapter 18 of

Section C, Computer Programs

Book 7, Automated Data Processing and Computations

Software support for probability calculations in three-part mineral resource assessments

Techniques and Methods 7-C18 


\section{U.S. Department of the Interior RYAN K. ZINKE, Secretary}

\section{U.S. Geological Survey James F. Reilly II, Director}

\section{U.S. Geological Survey, Reston, Virginia: 2018}

For more information on the USGS - the Federal source for science about the Earth, its natural and living resources, natural hazards, and the environment-visit https://www.usgs.gov or call 1-888-ASK-USGS.

For an overview of USGS information products, including maps, imagery, and publications, visit https://store.usgs.gov.

Any use of trade, firm, or product names is for descriptive purposes only and does not imply endorsement by the U.S. Government.

Although this information product, for the most part, is in the public domain, it also may contain copyrighted materials as noted in the text. Permission to reproduce copyrighted items must be secured from the copyright owner.

Suggested citation:

Shapiro, Jason, 2018, User's guide for MapMark4GUI—A graphical user interface for the MapMark4 R package: U.S. Geological Survey Techniques and Methods, book 7, chap. C18, 19 p., https://doi.org/10.3133/tm7c18.

ISSN 2328-7055 (online) 


\section{Contents}

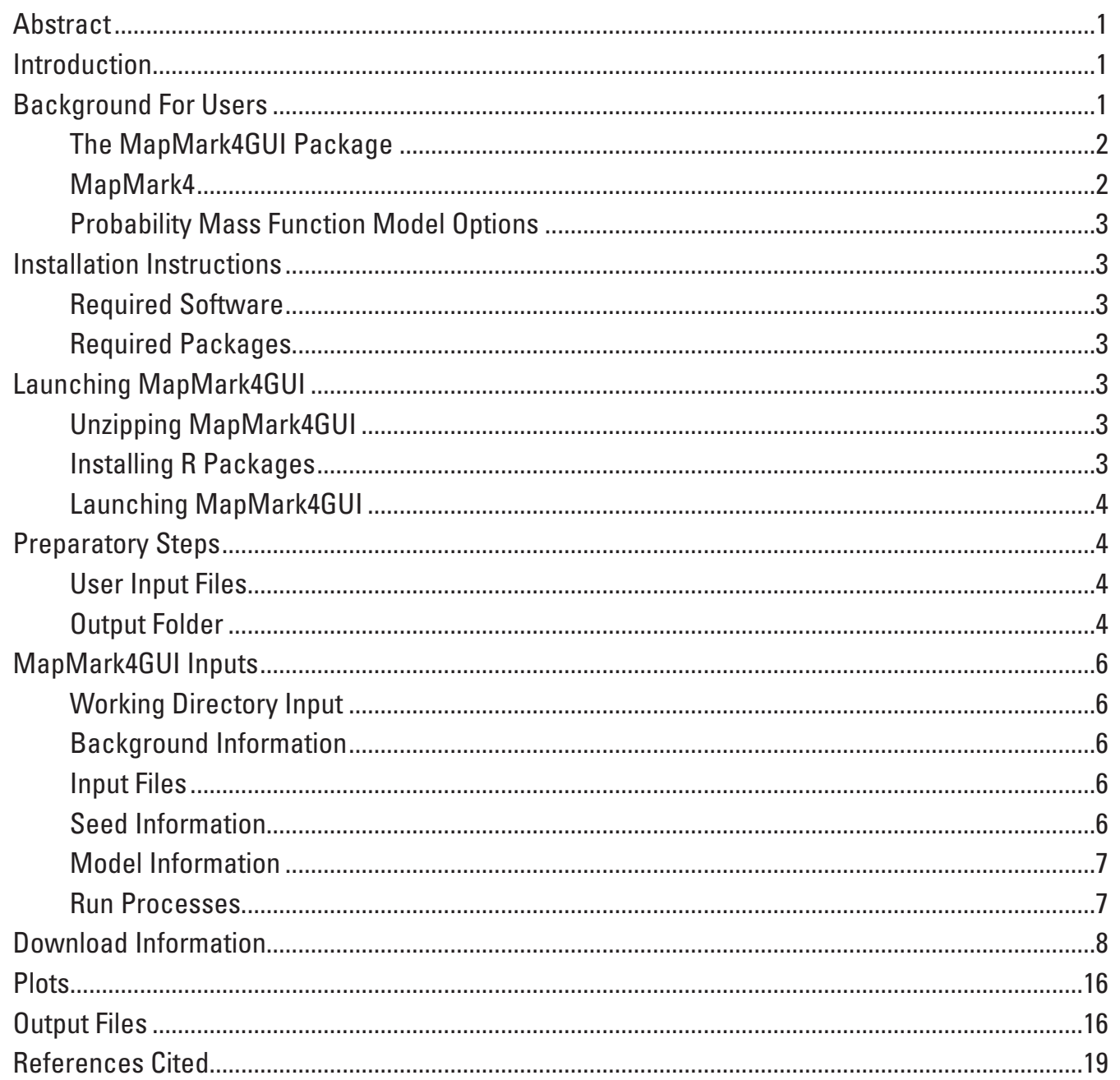




\section{Figures}

1. The MapMark4GUI dialog box with each of the input parameters filled in, including a random seed number and the MARK3 custom probability mass function model option with truncation and normal distribution

2. Graph showing MARK3 option probability mass function representing the number of undiscovered deposits in the permissive tract

3. Graphs showing negative binomial option probability mass function representing the number of undiscovered deposits in the permissive tract and probability mass function recast as elicitation percentiles and compared to the estimated numbers of undiscovered deposits

4. Graphs showing probability density function histogram that represents the ore tonnage in an undiscovered deposi and the corresponding cumulative distribution function

5. Histograms and cumulative distribution functions that were calculated from the probability density function that represents the grades.

6. Graphs showing univariate, marginal, probability density functions and univariate, marginal, complementary cumulative distribution functions for the total ore and mineral resource tonnages in all undiscovered deposits within the permissive tract

7. Matrix graphic showing the univariate and bivariate marginal distributions for the ore and mineral resource tonnages in all undiscovered deposits within the permissive tract

\section{Tables}

1. First six entries of the sample grade and tonnage model provided in the MapMark4GUI package

2. Example of the input estimates file format for the negative binomial probability mass function option

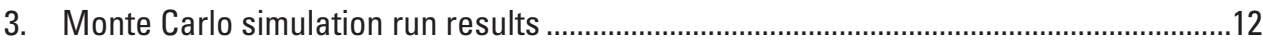

4. Simulation results aggregated by simulation run index...........................................14

5. Numbers of estimated undiscovered deposits and their relative probabilities...............14

6. Statistics for the probability mass function results ......................................................16

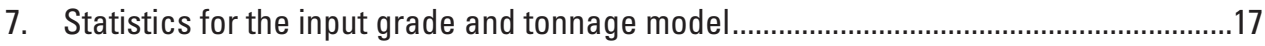

8. Statistics for the Monte Carlo simulation run ..................................................................17

9. Statistics for the contained resources......................................................................18 


\section{Abbreviations}

CRAN Comprehensive R Archive Network

CSV comma-separated values

EPS encapsulated PostScript

GTM grade and tonnage model

GUI graphical user interface

JPEG Joint Photographic Experts Group

PDF probability density function

PMF probability mass function

USGS U.S. Geological Survey 



\title{
User's Guide for MapMark4GUI_A Graphical User Interface for the MapMark4 R Package
}

\author{
By Jason Shapiro
}

\begin{abstract}
MapMark4GUI is an R graphical user interface (GUI) developed by the U.S. Geological Survey to support user implementation of the MapMark4 R statistical software package. MapMark4 was developed by the U.S. Geological Survey to implement probability calculations for simulating undiscovered mineral resources in quantitative mineral resource assessments. The GUI provides an easy-to-use tool to input data, run simulations, and format output results for the MapMark4 package. The GUI is written and accessed in the $\mathrm{R}$ statistical programming language. This user's guide includes instructions on installing and running MapMark4GUI and descriptions of the statistical output processes, output files, and test data files.
\end{abstract}

\section{Introduction}

MapMark4GUI is a graphical user interface (GUI) developed by the U.S. Geological Survey (USGS) to support implementation of the MapMark4 R package (Ellefsen, 2017a, b). The MapMark4 R package uses Monte Carlo simulation to estimate resources associated with estimates of undiscovered deposits defined by quantitative mineral resource assessment. MapMark4GUI is written in, and accessed by, the R statistical programming language. MapMark4GUI allows users to process the probability functions in MapMark4 (including estimates of undiscovered deposits and Monte Carlo simulation of undiscovered mineral resources), supports the analysis and visualization of the results, and creates a series of output files that summarize input parameters and quantitative results. The GUI does this by allowing users to parameterize and run the processes with a graphical dialog that has text boxes for user input and a set of buttons to run processes, alleviating the need to enter individual, specific R scripts. Use of the GUI decreases the chance for errors that may occur when entering specific R codes and therefore allows users to run MapMark4 with little knowledge of R coding. The GUI enables users to run probability assessments and Monte Carlo simulations for a range of study parameters. It also gives the user flexibility in selecting the probability and simulation processes and provides tables and graphs of the results. Users can easily transition between different process plots and output files to evaluate and compare results. In addition, the GUI provides statistics for the probabilities and simulation results, which may be used to support analysis of the results. The output files are generated by simply clicking on a set of buttons in the GUI dialog.

\section{Background For Users}

MapMark4 is the most recent software package developed by the USGS for simulation of undiscovered mineral resources in quantitative mineral resource assessments (Ellefsen, 2017a, b). The original program, known as MARK3, was used in an assessment of U.S. Forest Service wilderness tracts (Drew and others, 1986). Root and others (1992) described the MARK3 Monte Carlo computer simulator in detail. Both of these early versions were written in the FORTRAN programming language and run on mainframe computers. The FORTRAN code by Root and others (1992) was converted to the $\mathrm{C}++$ programming language and made available for (now obsolete) Microsoft Windows operating systems (Duval, 2000). That program was subsequently enhanced and published as EMINERS in 2004 (Bawiec and Spanski, 2012; Duval, 2012). Prior to the availability of MapMark4, all USGS quantitative mineral resource assessments used EMINERS or its predecessors. MapMark4 provides much greater flexibility and transparency for the user.

MapMark4GUI includes an option to use the MARK3 probability mass function (PMF) used in previous programs (Root and others, 1992; Singer and Menzie, 2010, appendix 2) or a negative binomial PMF for modeling the estimated numbers of undiscovered deposits. An important difference in MapMark4 relative to previous programs is that grade and tonnage models (GTMs) cannot include commodities that have incomplete data. Although GTMs include grade and tonnage values for the main commodity of interest, commodity grades for byproduct commodities may be incomplete. For example, the general porphyry copper GTM of Singer and others (2008) 
includes 422 tonnages and copper grades but has fewer grades for byproduct commodities such as molybdenum, gold, and silver. Users of MapMark4GUI should carefully consider which GTM to use and decide whether to impute missing grade values or omit byproduct commodities that are missing values. Guidelines for creating models and performing appropriate statistical tests are discussed in Singer and Menzie (2010). MapMark4GUI users are encouraged to explore the options available, carefully evaluate the data, and report estimated resources to no more than two significant figures.

\section{The MapMark4GUI Package}

MapMark4GUI runs the following MapMark4 processes: PMFs (NDepositsPmf, the probability that the number of undiscovered deposits is exactly equal to an integer value); probability density functions (PDFs; TonnagePdf and GradePdf, the probability of the continuous distribution of simulated ore tonnages and commodity grades); Monte Carlo simulation (Simulation), which samples the distributions of undiscovered deposits, ore tonnages, and commodity grades of estimated undiscovered resources; and the generation of the univariate and bivariate marginal distributions plots (Simulation Matrix) (Ellefsen, 2017a, b). Once the probabilities and simulations have been processed, MapMark4, implemented through the GUI, calculates and records a set of statistics regarding the deposit-scale tonnages and ore grades for the input GTM, the resulting probability functions, and the resulting simulated deposits. The recorded statistics include means, minimums, maximums, medians, standard deviations, percentiles, the probability of zero resources, and the probability that individual simulation results are greater than the mean of all simulation results.

The MapMark4GUI package includes the R-script code for the GUI, the script for automatic installation of the required R-script packages, and a set of sample user input files that can be used for a trial run of the GUI. The R-script code for the GUI is identified by the file name "MapMark4GUIRun.R." This script will launch and run MapMark4GUI. Prior to running the GUI script, the R packages need to be installed. This is done by running the installation script, identified by the file name "InstallPackages.R."

This report provides instructions for installing and using MapMark4GUI and further information regarding the process for estimating undiscovered resources and their probabilities using the GUI. The complete R script for MapMark4GUI is provided in the file "MapMark4GUIRun.R" that accompanies this report. The MapMark4GUI R software package is provided as a zipped folder that contains the following MapMark4GUI R scripts and supplementary sample input files:

- MapMark4GUIRun.R-The MapMark4GUI R run script used to launch and run the GUI.
- InstallPackages.R - The R script used to automatically install the R packages required to run MapMark4.

- MapMark4_1.0 (1).tar.gz-The MapMark4 R package that supports and implements the probability calculations and Monte Carlo resource simulations in R. This package is the current version as of November 2, 2017 (Ellefsen, 2017b).

- GTmodel.csv-A sample GTM input file in commaseparated values (CSV) format that can be used for a trial run of the GUI.

- Estimates.csv-A sample negative binominal probability estimates input file in CSV format that can be used for a trial run of the GUI.

- ReadMe.docx -A step-by-step guide for using MapMark4GUI.

- UserGuide_MapMark4GUI.pdf-A copy of this MapMark4GUI user's guide.

\section{MapMark4}

MapMark4GUI is an R-script GUI that supports user implementation of the MapMark4 software package.

MapMark4 is a Monte Carlo simulation program that performs resource estimates and is a replacement for the earlier MARK3 and EMINERS programs (Root and others, 1992; Bawiec and Spanski, 2012; Duval, 2012; Ellefsen, 2017a, b). MapMark4 is an R-script package published by the USGS (Ellefsen, 2017a, b). The MapMark4 R package can be downloaded from https://doi.org/10.3133/tm7C14 (Ellefsen, 2017b).

To learn more about the underlying MapMark4 processes and calculation techniques, see the corresponding reports: "Probability Calculations for Three-Part Mineral Assessments" (Ellefsen, 2017a) and "User's Guide for MapMark4-An $R$ Package for the Probability Calculations in Three-Part Mineral Resource Assessments" (Ellefsen, 2017b).

The three-part method of quantitative resource assessment was described by Singer and Menzie (2010). The first part of the method uses known tonnage and grades at well-characterized deposits to describe the range of possible resources contained in undiscovered deposits. The GTM is one of the input files used by MapMark4 (Singer and Menzie, 2010, p. 77-104). The second part of the method is the delineation of a geographic area where the existence of the deposit types under consideration is possible; this area is called the permissive tract or mineral resource map (Singer and Menzie, 2010, p.105-117). The third part of the assessment method is a probabilistic estimate of the number of undiscovered deposits that could be in the permissive tract elicited at different confidence levels (Singer and Menzie, 2010, p. 118-147). These estimates are user input used byMapMark 4 to calculate 
the PMF, which provides an estimate of numbers of undiscovered deposits at different confidence levels. The PMF for the number of undiscovered deposits is then used as an input to sample the GTM, providing quantitative estimates of contained resources that could be present in the permissive tract (Ellefsen, 2017a, b).

\section{Probability Mass Function Model Options}

A PMF is used to estimate the number of undiscovered deposits in the permissive tract (Ellefsen, 2017a, p. 5). MapMark4GUI supports the implementation of the PMF calculation using one of two options. The first being MapMark4's negative binominal option, which estimates the number of undiscovered deposits in the tract using a negative binominal distribution (Ellefsen 2017a, p. 5). More information regarding this method can be found in Ellefsen (2017a). When running this option, the MapMark4 process requires an input file with deposit number estimates for three probability levels - N90, N50, and N10, where Np is the number of undiscovered deposits at the specific probability level $\mathrm{p}$ (Ellefsen, 2017a, p. 2). More information about this input file is detailed in the "User Input Files" section.

The other option is the MARK3 option, which is a custom function that allows the user to enter the deposit number estimates at five probability levels. This option is recommended for use with permissive tracts in which the probability of undiscovered deposits is low and the N90 or the N50 and N10 estimates are zero. The MARK3 PMF option uses the algorithm given in appendix 2 of Singer and Menzie (2010), which is an extension to the Root and others (1992) algorithm. The MARK3 PMF algorithm estimates deposit-number probabilities and determines an expected number of deposits using a set of user-entered N90, N50, N10, N05, and N01 estimated percentiles (Singer and Menzie, 2010). Five levels of expected deposit numbers must be entered; if estimates were not made at the N01 or at both the N01 and N05 levels, the user must enter the values of the previous probability level to complete all five levels of estimates. Either PMF option can be selected using the drop down menu under the "Model Information" section of the MapMark4GUI dialog.

\section{Installation Instructions}

\section{Required Software}

MapMark4GUI was developed to be used with an R console, software that can read and process $\mathrm{R}$ scripts. To launch MapMark4GUI, installation of an R console is required. It is recommended that you use the Comprehensive R Archive Network (CRAN) 64- or 32-bit R console to run the GUI.
To download the R console for Windows, go to the CRAN R project website (https://cran.r-project.org/bin/windows/base). At the website, click on the "Download R for Windows" option and follow the instructions provided at the website to complete the installation. The version available for download will be the current release.

\section{Required Packages}

When working with $\mathrm{R}$, many processes are dependent on external packages that can be installed and uploaded to the $\mathrm{R}$ console for future work. MapMark4GUI requires a selection of $\mathrm{R}$ packages to run. $\mathrm{R}$ packages need only be installed once so long as the packages have not been removed or edited; however, packages can be reinstalled multiple times without errors.

A script provided in the GUI package (InstallPackages.R) installs the required dependent packages automatically. The following R packages are required for the GUI to run:

- ggplot2

- gWidgets

- gWidgetstcltk

- dplyr

- compositions

- mvtnorm

- ks

- MapMark4 (provided in the package file)

\section{Launching MapMark4GUI}

\section{Unzipping MapMark4GUI}

The MapMark4GUI package is downloaded as a zip file. The zip file includes a folder with the install script, run script, and sample input files. To launch MapMark4GUI, the GUI file will need to be unzipped. This can be done by right clicking the zip file and then clicking "Extract." Unzip the file to a folder where it can be conveniently accessed.

\section{Installing R Packages}

Before launching MapMark4GUI, the R console must be activated on your computer. This involves the installation of required and dependent $\mathrm{R}$ packages. In the $\mathrm{R}$ console, open the installation script to start the installation run by clicking "File" on the top menu and then clicking "Open script." Navigate 
to the location where the GUI folder was unzipped and select "InstallPackages.R." Clicking on the file will open the installation script. Click "Edit" on the top menu and then click "Run all" to run the complete installation script automatically. Three popup windows will be displayed during this process, and each will wait for user input before continuing the installation run. The first dialog asks whether the user would like to create a new personal library at the location indicated. Click "Yes" to create a library inside the R-console directory in which to save the required packages, allowing use of the GUI without installing the packages each time $\mathrm{R}$ is opened. The second dialog asks for the CRAN mirror from which to download the R packages. The mirror US_CA1 was tested and worked successfully for MapMark4GUI. The final dialog will be a browser dialog that asks the user to identify the file containing the MapMark4 package (MapMark4_1.0 (1).tar.gz). Find and click on the MapMark4 package (the "MapMark4 1.0 (1). tar.gz" file will be highlighted), then click "Open" to install the MapMark4 software. A copy of the current MapMark4 package is provided in the supplementary MapMark4GUI folder. Once installation is complete, MapMark4GUI can be launched.

\section{Launching MapMark4GUI}

To start the GUI, click "File" in the open R console and then click "Open script." Navigate to and select the MapMark4GUI file (MapMark4GUIRun.R) to open the R script for the GUI. Next, click "Edit" in the R console menu and select "Run all." The GUI script will run and launch the GUI dialog box for MapMark4GUI (MapMark Inputs dialog box). The MapMark Inputs dialog box will be created as the script runs. The script is complete when the buttons below "Plots" are available (fig. 1).

\section{Preparatory Steps}

\section{User Input Files}

To enable MapMark4GUI to dynamically run MapMark4 on the basis of user input, a GTM user input file is required. If the negative binominal PMF option is used, an input file of deposit number percentile estimates is also required. If the MARK3 option is selected, the user must enter the deposit number percentile estimates in the MapMark Inputs dialog box.

A GTM is a CSV data table of known deposits in the permissive tract that includes the grades and tonnages of each commodity being tested and is required to run MapMark4GUI. An example GTM (GTmodel.csv) is provided in the MapMark4GUI package (table 1). The first two columns are required, unique identifiers for the individual deposits in the model. The unique identifiers are supplemental information to identify individual deposits in the model. The first two columns can be given any name. In the example given, the first column is each deposit's coded ID (Coded_ID), and the second column is each deposit's name (Name). The next columns provide the deposit data. The third column lists the ore tonnage in metric tons and must be named "Ore." The remaining columns list the commodity grades in percent units, and the column headings must be the commodity abbreviations without percentage signs. The file may be prepared in Microsoft Excel and saved in the required CSV format. Missing grades are not permitted. If grade values for commodities are missing, it is up to the user to use an appropriate method to estimate missing values. The GTM should include at least 20 deposits for the MapMark4 processes to successfully run using the GUI.

If the negative binomial PMF option is selected, a second user input file is required. This file is a CSV table of estimates of the number of undiscovered deposits (table 2). This CSV table includes an estimator identifier, an optional weighting value for each estimator, and estimates of the number of undiscovered deposits at the 90-, 50-, and 10-percentile levels (Ellefsen, 2017a, p. 3-4). The estimates table can have one estimate or a weighted set of estimates for multiple estimators. A sample estimates file that has a single estimate row (estimates.csv) is provided in the MapMark4GUI package to support a trial run of the GUI. The example shown in table 2 is for multiple estimators. Each row in the input estimates file (for example, table 2) represents an individual estimate with an assigned weight that provides a measure of degreeof-importance or influence for each individual estimate. Weight values range from 0 ( 0 percent degree-of-importance or influence) to 1 (100 percent degree-of-importance or influence). See Zientek and others (2014, p. 23-24) for a discussion of weighting estimates. In cases where a single consensus estimate is entered, the weight should be set to a default value of 1 . Estimates for the number of undiscovered deposits can be made only for the 90-, 50-, and 10-percentile levels. The MARK3 PMF model option must be used if additional percentile levels are desired.

\section{Output Folder}

The first user input in MapMark4GUI is setting the working directory. This is the folder where outputs from the GUI processes will be saved. Running MapMark4GUI will output 14 files for each simulation run. It is recommended that you create a new output folder for each simulation run to aid organization. To set the working directory, click the "browse" button in the "Working Directory Input" section of the MapMark Inputs dialog, then click "Make New Folder," or right-click the folder list and click "Create New Folder." It is recommended that you give the output folder a unique, short name that gives a useful description of the run to help organize the different process runs. 
Figure 1. The MapMark4GUI dialog box with each of the input parameters filled in, including a random seed number and the MARK3 custom probability mass function (PMF) model option with truncation and normal distribution.
MapMark Inputs

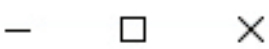

Working Directory Input

\begin{tabular}{ll|l|l|l|l|l|l|l|}
\hline Working Directory: & frowse & Set Working Directory
\end{tabular}

Background Information

Tract ID: Tract1 Run ID: Run1

Input Files

GTM Input: (GT deposits $>=20$ )

F:/RWork/GTmodel.csv browse

Estimates file for NegBinomial Type:

\begin{tabular}{|l|l}
\hline Select a file... & browse
\end{tabular}

Seed Information

Create Random Seed Set Specific Seed 72

Model Information

PMF model:

MARK3

Enter the N90, N50, N10, N05, and N01 percentiles [MARK3 Option Only] If no estimates were made at N05 and N01, use the value from N10

01234

Truncate?:

TRUE

Distribution type: (kde should be only used if the GTM has $>50$ deposits)

normal

Run Processes

\section{\begin{tabular}{|l|l|l|l|l|} 
1. Run Pmf & 2. Run TonPdf & 3. Run GradePdf & 4. Run Simulation & 5. Run Sim Matrix
\end{tabular}}

Download Information

Download Parameters \& Summary Download PMF Stats Download Sim and GTM Stats

Plots

\begin{tabular}{|l|l|l|l|l|l|l|l} 
Plot PMF & Plot Ton PDF Plot Grade PDF Plot Simulation Results Plot Sim Matrix \\
\hline
\end{tabular}


Table 1. First six entries of the sample grade and tonnage model provided in the MapMark4GUI package.

[The grade and tonnage model is a table listing known deposits. The first two columns provide unique identifiers for the datasets that include the deposit identifier (Coded ID) and name (Name). The third column lists the ore tonnages in metric tons (Ore), and the remaining columns list the commodity grades in percent units. $\mathrm{Cu}$, copper; Mo, molybdenum; Au, gold; Ag, silver]

\begin{tabular}{lllllll}
\hline \multicolumn{1}{c}{ Coded_ID } & \multicolumn{1}{c}{ Name } & Ore & Cu & Mo & Au & Ag \\
\hline $142 \mathrm{pCu} 8704$ & Fengshandong & $1.05 \mathrm{E}+08$ & 0.38 & 0.05 & 0.000037 & 0.002 \\
$142 \mathrm{pCu} 8704$ & Chengmenshan & $4.09 \mathrm{E}+08$ & 0.75 & 0.047 & 0.000024 & 0.00099 \\
$150 \mathrm{pCu} 6004$ & Státiavnica Group & $2.10 \mathrm{E}+08$ & 0.35 & 0.001 & 0.000019 & 0.000952 \\
$003 \mathrm{pCu} 9004$ & Continental/Butte & $5.22 \mathrm{E}+09$ & 0.673 & 0.028 & 0.0000042 & 0.00086 \\
$142 \mathrm{pCu} 8502$ & Kyzyltu & $6.58 \mathrm{E}+07$ & 0.48 & 0.015 & 0.00002 & 0.000656 \\
$142 \mathrm{pCu} 8003 \mathrm{~b}$ & Kounrad & $6.37 \mathrm{E}+08$ & 0.589 & 0.011 & 0.000019 & 0.000628 \\
\hline
\end{tabular}

Table 2. Example of the input estimates file format for the negative binomial probability mass function option.

[The "Name" column lists the identifiers of individual estimators. The "Weight" column assigns the degree-of-importance for the estimate; higher values indicate greater weight, and lower values indicate less weight. Weight values range from 0 to 1 . The remaining columns give the numbers of undiscovered deposits estimated at the 90-, 50-, and 10-percentile levels]

\begin{tabular}{lcccc}
\hline Name & Weight & N90 & N50 & N10 \\
\hline A & 1 & 1 & 1 & 1 \\
B & 0.5 & 2 & 3 & 4 \\
C & 1 & 1 & 1 & 2 \\
D & 0.25 & 3 & 5 & 7 \\
\hline
\end{tabular}

\section{MapMark4GUI Inputs}

MapMark4GUI launches a dialog that accepts user input for the process parameters required to run the probability functions and Monte Carlo simulation (fig. 1). The GUI also allows the user to create and download a variety of statistics on the simulation results. The dialog features five sections for user input parameters. The next sections discuss the input parameters and instructions on completing the entries.

\section{Working Directory Input}

The first entry in the MapMark Inputs dialog sets the working directory. This entry points the GUI to the output folder directory where the output files of the probability functions and simulation will be saved. The output and input files can be stored in separate folders to aid organization. The first step is to click the "browse" button and navigate to the output folder. Click on the output folder name and click "OK" or "Select folder" in the browse folder dialog to confirm the selected folder. In the MapMark Inputs dialog, click on "Set Working Directory" to change the R working directory to the selected output folder. The address of the working directory is printed in the $\mathrm{R}$ console. To verify that the working directory has been changed correctly, check the printout in the console.

\section{Background Information}

The next input parameters are identifiers for the tract name and run name. The Tract ID is the name of the assessment tract, which is a string type input that allows integers and text (short names are desirable). To enter the Tract ID, click in the text field box, delete the current entry, and type in the new Tract ID. The next input is the Run ID (also a string input), which is the name of the run simulation. To enter the Run ID, delete the current entry and enter the new Run ID in the text field box. All output files will incorporate the Run ID into their respective names. In this user's guide, italic in output file names indicates where the user-entered Run ID will be incorporated (for example, RunIDpmfPlot.eps).

\section{Input Files}

The next entry in the dialog identifies the locations of the user input CSV files. The GTM file is required for all simulation runs, and the estimates file is required if the negative binomial (NegBinomial) PMF model option is selected. The MapMark4 reports by Ellefsen $(2017 \mathrm{a}, \mathrm{b})$ provide more details on the input file design, input file reasoning, and implementation of the MapMark4 processing steps. Sample files (GTmodel.csv and estimates.csv) are included in the supplementary folder as examples of input files that can be used for trial runs of the GUI. To select an input file, click on the "browse" button to the right of the appropriate file input box and navigate to the folder where the GTM and estimates files are located. Choose the appropriate file and click "OK." Note that the estimates file is required only if using the negative binomial PMF model option. If using the MARK3 PMF model option, leave the estimates file input box blank and proceed to the next section.

\section{Seed Information}

The fourth parameter section specifies the seed number to be used in the Monte Carlo simulation run. The seed number is 
a positive integer. In the MapMark Inputs dialog, the user has a choice to create a random seed number or enter a specific seed number. Click the "Create Random Seed" button to generate a random integer number between 1 and 100. The seed number will appear to the right of the "Set Specific Seed" button. The "Create Random Seed" button can be clicked repeatedly to change the random seed selection. Each time a random seed number is created, the new number will appear to the right of the previous seed number. To enter a specific seed number instead of generating a random number, click on the "Set Specific Seed" button and enter the number in the blank box that appears to the right of the button. Using consistent seed numbers will reproduce the same simulation results (Ellefsen, 2017b, p. 5).

\section{Model Information}

The next section of the input dialog specifies the parameters for the PMF model, including the model type, estimated numbers of undiscovered deposits (if the MARK3 option is used), the truncation option, and the distribution type.

The PMF model options are named "MARK3" and "NegBinomial." To make a choice, click on the drop down menu labeled "Select a model" and click on the desired option. Selection of the negative binomial option requires the user to input an estimates file (discussed in the "User Input Files" section). When "NegBinomial" is selected, the text box for the estimated numbers of undiscovered deposits has a default entry of "NA" for not applicable. For the MARK3 option, enter the numbers $(\mathrm{N})$ of the undiscovered deposits estimated at the various percentile levels-N90, N10, N05, and N01. Five percentile input values are required. If three non-zero percentiles - N90, N50, and N10 - are estimated, repeat the $\mathrm{N} 10$ value for N05 and N01. If four non-zero percentilesN90, N50, N10, and N05 - are estimated, repeat the N05 value for N01. The numbers should be separated by spaces with no commas (for example, 01234 ).

The truncation option dropdown menu allows selection of either "TRUE" or "FALSE." If "TRUE" is selected, MapMark4 truncates the grade and ore tonnage PDF results between the lowest and highest values in the GTM (Ellefsen, 2017b). If "FALSE" is selected, no truncation will occur and the simulated deposit grade and tonnage values will not be constrained within the range of the GTM values. "TRUE" is the recommended option.

The distribution type dropdown menu allows the user to specify how the grade and ore tonnage PDF is estimated. The two choices are "normal" (normal distribution) and "kde" (kernel density function). If the normal distribution option is used, the data are log-transformed and a normal distribution is used to develop the PDF. This is the default recommended option (Ellefsen 2017a, p. 3). The kernel density option uses a Gaussian kernel density estimate to develop the PDF (Ellefsen, 2017a, p. 3). The kernel density option is not recommended unless the GTM has approximately 50 or more deposits and may fail if there are fewer than 50 deposits (Ellefsen, 2017b, p. 5).

\section{Run Processes}

MapMark4GUI supports the implementation of a range of MapMark4's probability and simulation processes. Once all input parameters have been entered in MapMark4GUI's inputs dialog, the probability analyses and Monte Carlo simulation can be started by clicking sequentially on the buttons in the "Run Processes" section. Each process button will be highlighted in blue while the process is running. The next process cannot be selected until the previous process has ended, as indicated by the appearance of the plots on the $\mathrm{R}$ console screen. The MapMark4GUI process steps are based on the MapMark4 probability calculation groups called "software classes" (Ellefsen, 2017b, p. 1). The following MapMark4 processes can be run in sequential order in MapMark4GUI:

1. NDepositsPmf - Creates the PMF for the number of undiscovered deposits.

2. TonnagePdf - Creates the PDF for the undiscovered deposit ore tonnage.

3. GradePdf - Creates the PDF for the commodity grades.

4. Simulation-Runs the Monte Carlo simulation.

5. Simulation Matrix - Creates a matrix of each of the simulation's resulting univariate and bivariate marginal distributions.

The first MapMark4 process, NDepositsPmf, generates the PMF for the number of undiscovered deposits. This process builds a PMF model for the number of undiscovered mineral deposits at the various percentile estimates (Ellefsen, 2017b, p. 2) and is initiated by clicking the "Run Pmf" button. The process generates a PMF plot on the R console screen and writes the plot to the output folder as encapsulated PostScript (EPS) and Joint Photographic Experts Group (JPEG) files (RunIDpmfPlot.eps and RunIDpmfPlot.jpg). The plot shows the number of undiscovered deposit estimates for various probability levels. The negative binominal PMF plot includes the elicitation probabilities and compares the estimates with the user-provided estimates for the 90-, 50-, and 10 -percentile levels. Examples of the PMF result plots for the MARK3 and negative binomial options are shown in figures 2 and 3 , respectively.

The second MapMark4 process, TonnagePdf, creates a PDF for the undiscovered deposit ore tonnage by considering the probability and uncertainty of the tonnage (Ellefsen, 2017 b, p. 2). This process is initiated by clicking the "Run TonPdf" button. A PDF plot is generated on the R console screen and written to the output folder as EPS and JPEG files (RunIDTonPdfPlot.eps and RunIDTonPdfPlot.jpeg). The PDF plot shows ore tonnage as a density histogram and as a probability curve (fig. 4). 
Figure 2. MARK3 option probability mass function representing the number of undiscovered deposits in the permissive tract.

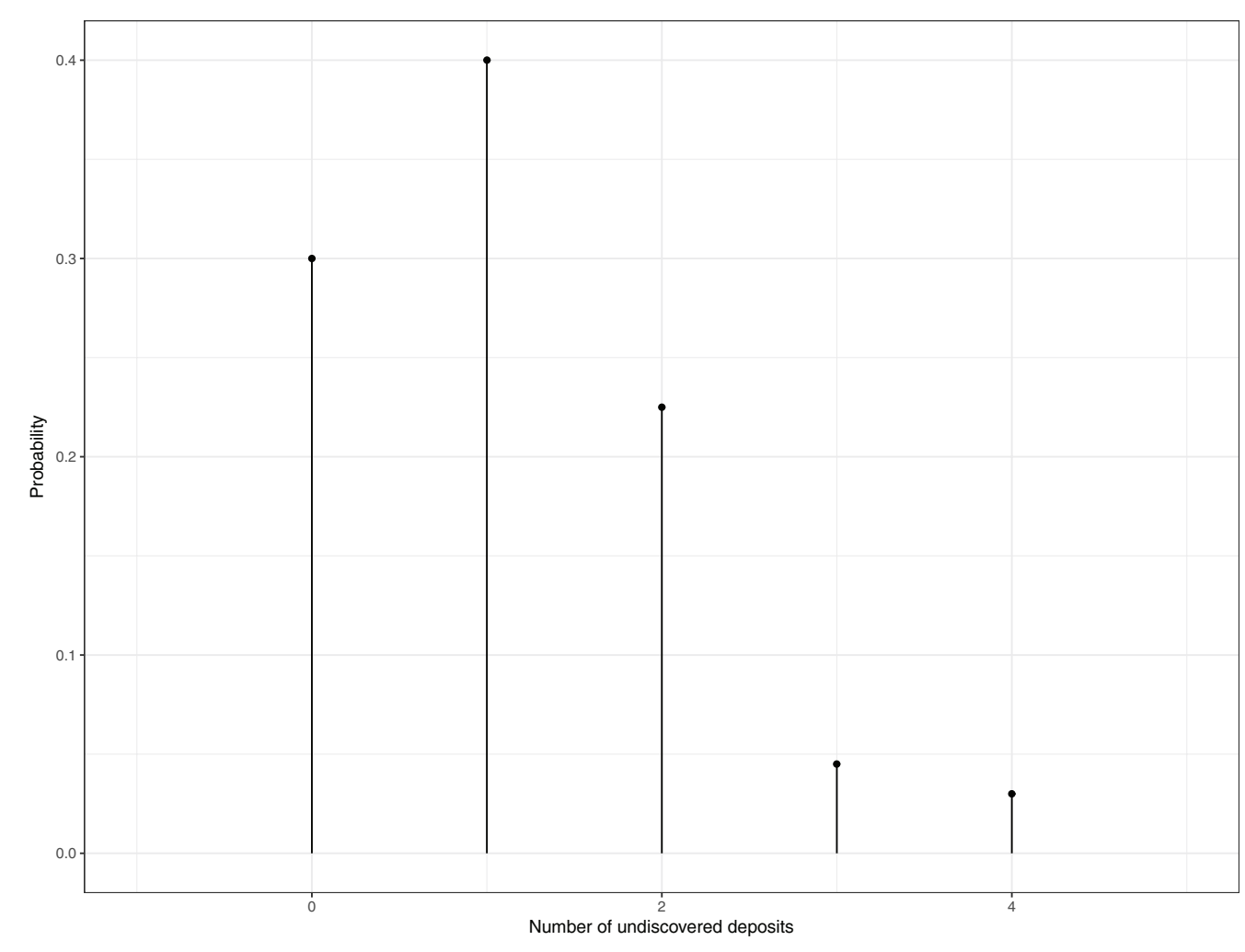

that includes the ore tonnages and contained commodity tonnages (table 4).

The fifth and final process, Simulation Matrix, generates a marginal plot matrix of the simulation's univariate and bivariate marginal distributions of the ore and commodity tonnages (Ellefsen, 2017b, p. 13). The process is initiated by clicking the "Run SimMatrix" button. The matrix of plots is generated on the R console screen (fig. 7) and written to the output folder as EPS and JPEG files (RunIDSimMatrixPlot.eps and RunIDSimMatrixPlot.jpg).

\section{Download Information}

MapMark4GUI runs several statistical processes and generates output files for the probability calculations and simulation results. Each of the statistical processes is initiated by sequentially clicking the buttons in the "Download Information" section of the MapMark Inputs dialog.

Clicking the "Download Parameters \& Summary" button generates a CSV file (RunID_01_InputParameters.csv) of all the input parameters entered into the GUI by the user and a text file of statistics comparing the PDFs for ore tonnage and commodity grades with the actual values in the GTM (RunID_ Summary.txt).

Clicking the "Download PMF Stats" button processes the numbers of undiscovered deposits estimated at various percentile levels and calculates their relative probabilities for the PMF model (RunID_03_PMF_Probs.csv) using the MapMark4 NDepositsPmf process (table 5). 

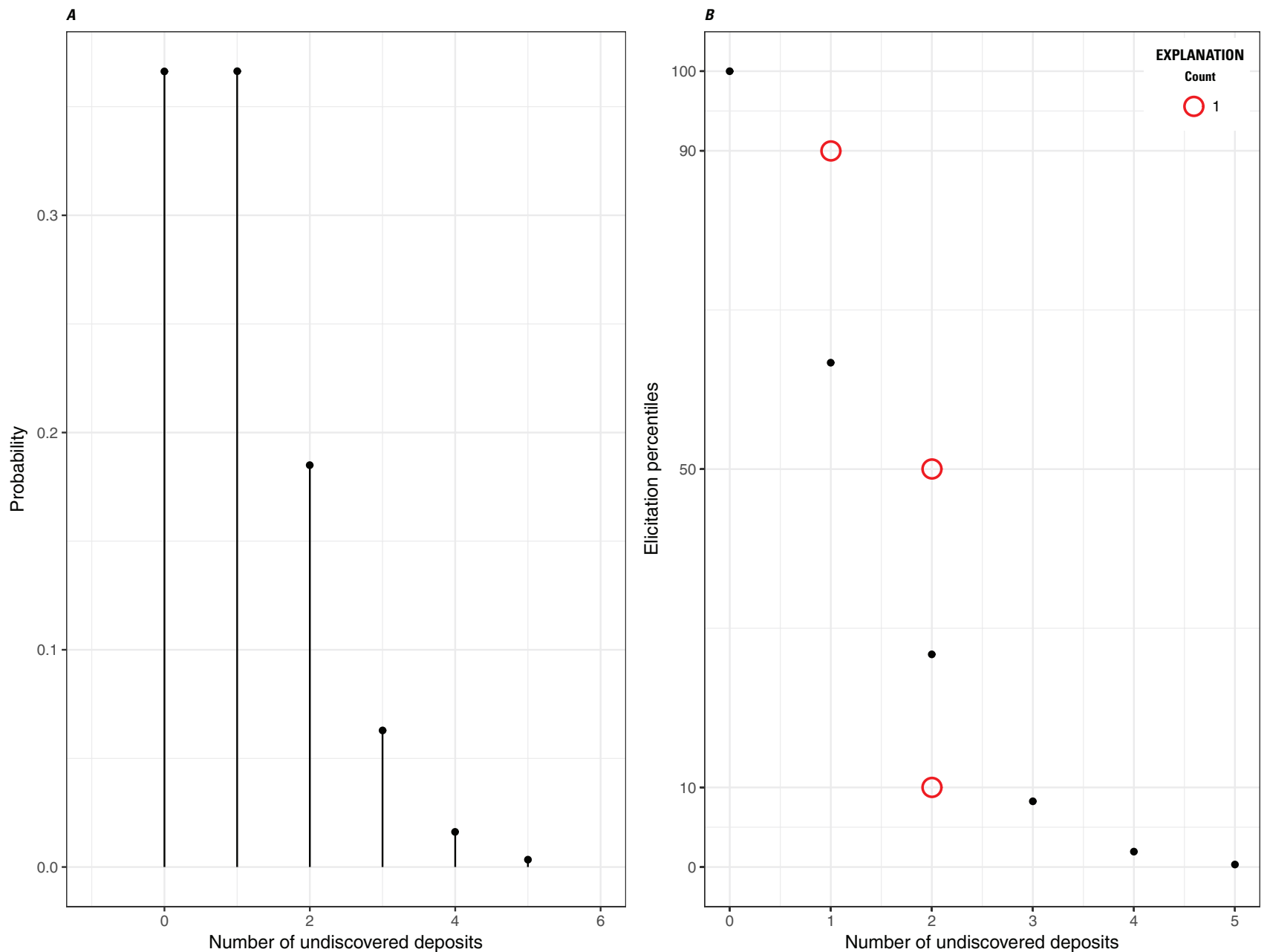

Figure 3. A, Negative binomial option probability mass function representing the number of undiscovered deposits in the permissive tract. $B$, Probability mass function recast as elicitation percentiles (black dots) and compared to the estimated numbers of undiscovered deposits (red circles). The size of a red circle indicates how many assessment team members picked the same number of undiscovered deposits. In this example, only one set of estimates was entered. 

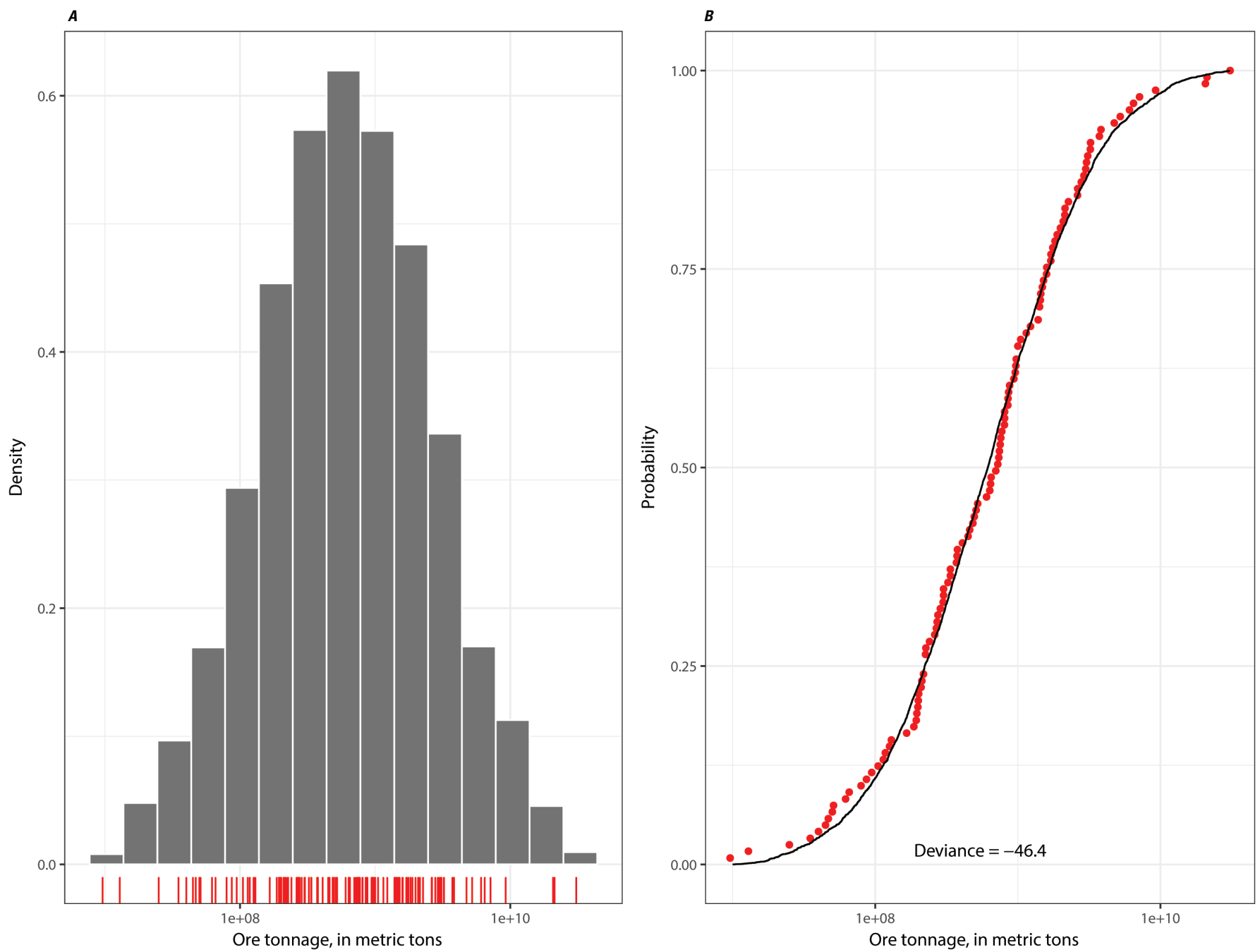

Figure 4. A, Probability density function histogram that represents the ore tonnage in an undiscovered deposit. The red vertical lines at the bottom represent the ore tonnages from the grade and tonnage model. $B$, The corresponding cumulative distribution function (solid black line). The red dots constitute the empirical cumulative distribution function for the ore tonnages from the grade and tonnage model. 

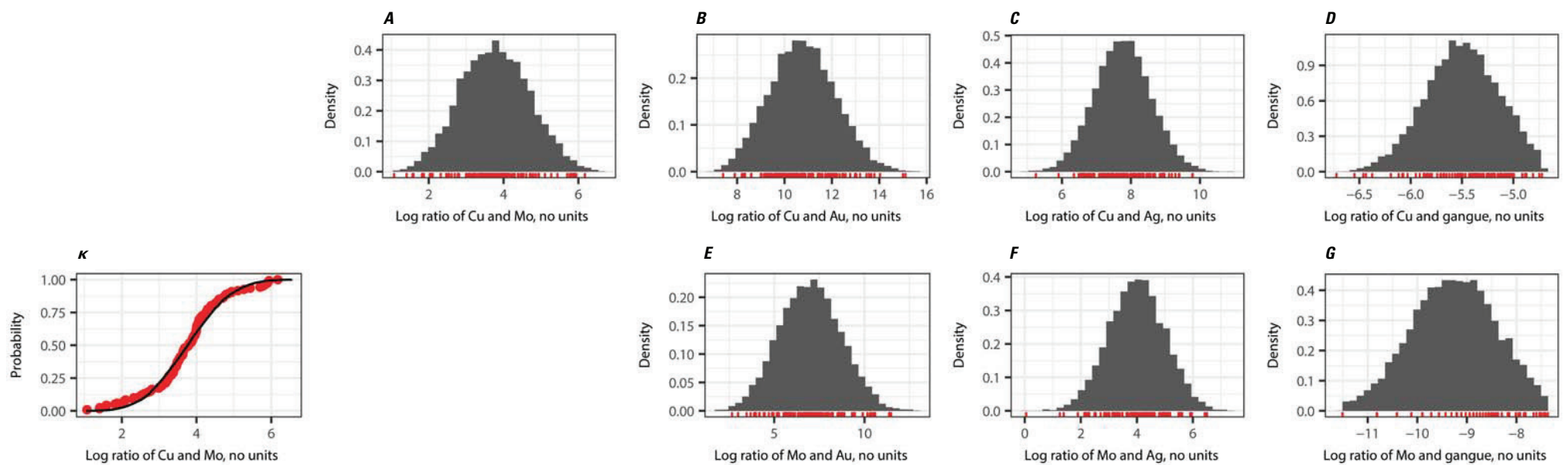

Log ratio of $\mathrm{Cu}$ and $\mathrm{Mo}$, no units

Log ratio of Cu and Au, no units

Log ratio of $\mathrm{Cu}$ and $\mathrm{Ag}$, no units

Log ratio of $\mathrm{Cu}$ and gangue, no units
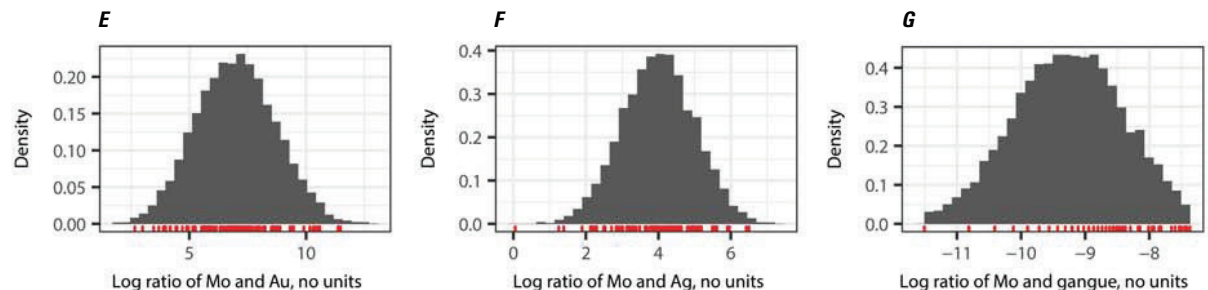

Log ratio of Mo and Au, no units

Log ratio of Mo and Ag, no units

Log ratio of Mo and gangue, no units

M
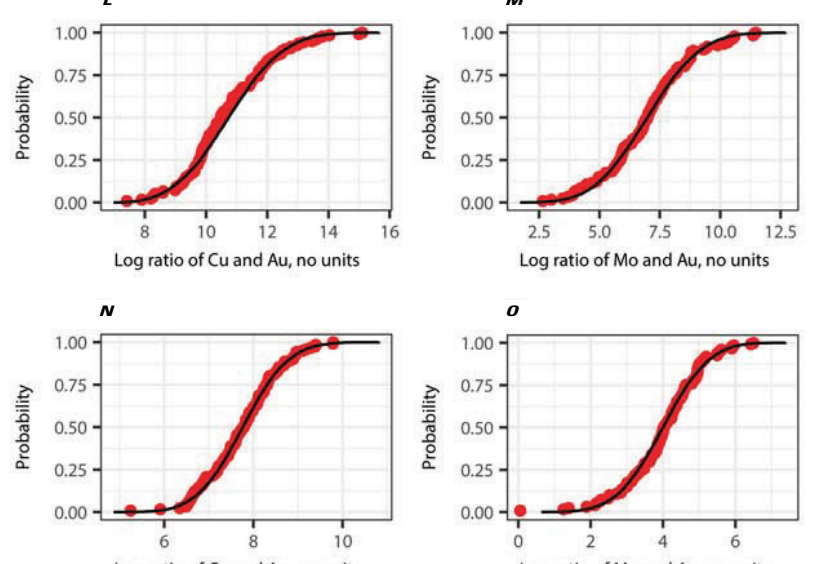

Log ratio of $\mathrm{Cu}$ and $\mathrm{Ag}$, no units

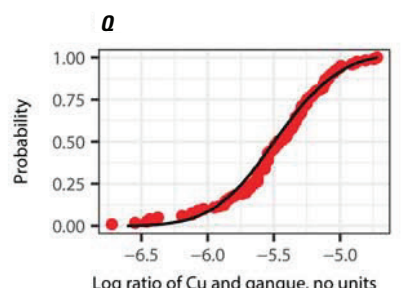

Log ratio of $C u$ and gangue, no units

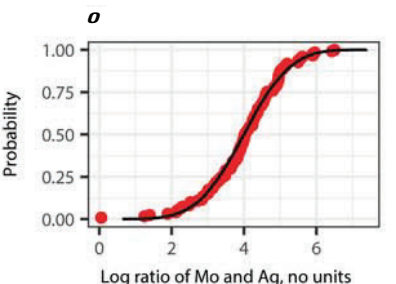

Log ratio of Mo and Ag, no units

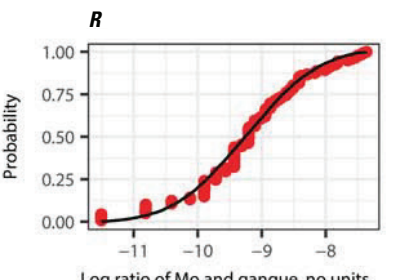

Log titio $\mathrm{mo}$ and gange no units

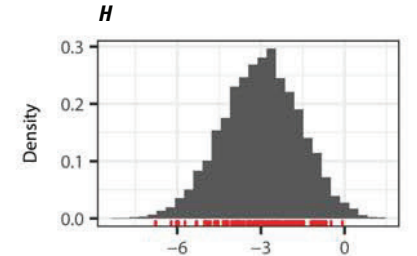

Log ratio of Au and Ag, no units

$P$
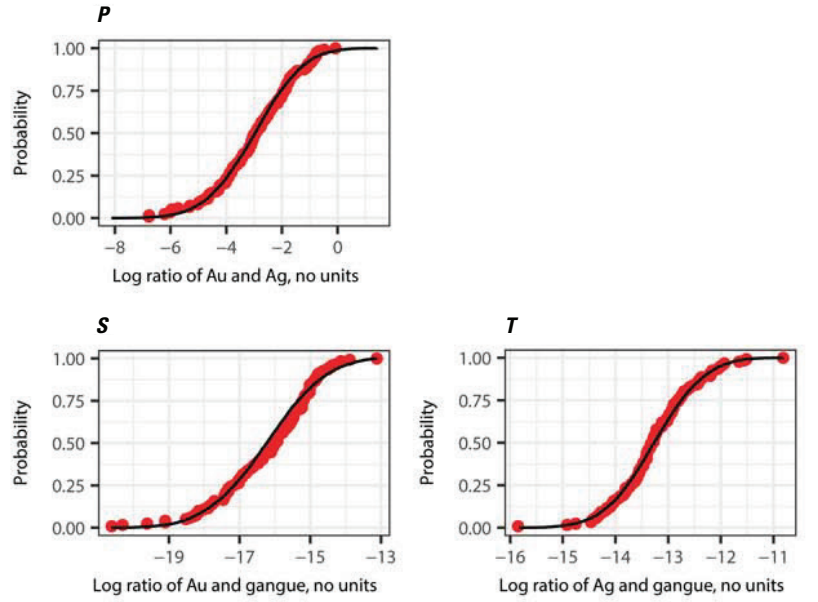
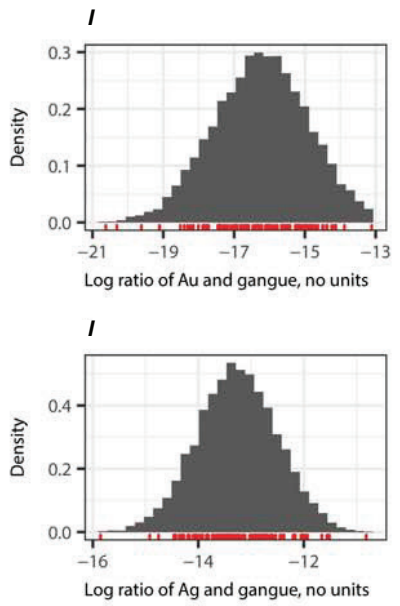

Log ratio of Ag and gangue, no units

Figure 5. Histograms $(A-J)$ and cumulative distribution functions $(K-T)$ that were calculated from the probability density function that represents the grades. In the histograms, the vertical red lines at the bottom of each plot represent the log-ratios calculated from the grade and tonnage model. In the cumulative distribution functions, the red dots constitute empirical cumulative distribution functions for the log-ratios calculated from the grade and tonnage model. This example uses a porphyry copper grade and tonnage model that includes the commodities copper (Cu), molybdenum (Mo), gold (Au), and silver $(\mathrm{Ag})$. The source is a subset of data from the Singer and others (2008) porphyry copper model that is not missing values. 
Table 3. Monte Carlo simulation run results.

[The "Ore MetricTons" column gives the ore tonnages in metric tons. The grades of each commodity for each simulation run deposit are listed in percent units in columns "Cu pct" through "gangue pct," and the in-place amounts of resources for each commodity included in the grade and tonnage model are listed in metric tons in columns "Cu_MetricTons" through "Ag_MetricTons." Cu, copper; Mo, molybdenum; Au, gold; Ag, silver]

\begin{tabular}{|c|c|c|c|c|c|c|c|c|c|c|c|c|c|}
\hline Run & $\begin{array}{l}\text { Simulation } \\
\text { Index }\end{array}$ & $\begin{array}{c}\text { Number of } \\
\text { Deposits }\end{array}$ & $\begin{array}{c}\text { Sim.Deposit. } \\
\text { Index }\end{array}$ & $\begin{array}{c}\text { Ore_Metric } \\
\text { Tons }\end{array}$ & Cu_pct & Mo_pct & Au_pct & Ag_pct & gangue_pct & $\begin{array}{c}\text { Cu_Metric- } \\
\text { Tons }\end{array}$ & $\begin{array}{c}\text { Mo_Metric- } \\
\text { Tons }\end{array}$ & $\begin{array}{c}\text { Au_Metric- } \\
\text { Tons }\end{array}$ & $\begin{array}{c}\text { Ag_Metric- } \\
\text { Tons }\end{array}$ \\
\hline 1 & 1 & 4 & 1 & $4.77 \mathrm{E}+08$ & $3.37 \mathrm{E}-01$ & $5.02 \mathrm{E}-03$ & $1.78 \mathrm{E}-05$ & $1.78 \mathrm{E}-04$ & $9.97 \mathrm{E}+01$ & $1.61 \mathrm{E}+06$ & $2.40 \mathrm{E}+04$ & $8.50 \mathrm{E}+01$ & $8.49 \mathrm{E}+02$ \\
\hline 2 & 1 & 4 & 2 & $1.96 \mathrm{E}+09$ & 4.33E-01 & $1.46 \mathrm{E}-02$ & $6.41 \mathrm{E}-06$ & $1.95 \mathrm{E}-04$ & $9.96 \mathrm{E}+01$ & $8.51 \mathrm{E}+06$ & $2.88 \mathrm{E}+05$ & $1.26 \mathrm{E}+02$ & $3.83 \mathrm{E}+03$ \\
\hline 3 & 1 & 4 & 3 & $8.92 \mathrm{E}+08$ & $6.16 \mathrm{E}-01$ & $1.11 \mathrm{E}-02$ & $1.91 \mathrm{E}-05$ & $2.66 \mathrm{E}-04$ & $9.94 \mathrm{E}+01$ & $5.50 \mathrm{E}+06$ & $9.91 \mathrm{E}+04$ & $1.70 \mathrm{E}+02$ & $2.38 \mathrm{E}+03$ \\
\hline 4 & 1 & 4 & 4 & $1.18 \mathrm{E}+09$ & $4.19 \mathrm{E}-01$ & $1.55 \mathrm{E}-03$ & $3.33 \mathrm{E}-05$ & $1.27 \mathrm{E}-04$ & $9.96 \mathrm{E}+01$ & $4.93 \mathrm{E}+06$ & $1.82 \mathrm{E}+04$ & $3.92 \mathrm{E}+02$ & $1.49 \mathrm{E}+03$ \\
\hline 5 & 2 & 4 & 1 & $8.51 \mathrm{E}+08$ & $4.71 \mathrm{E}-01$ & $9.07 \mathrm{E}-03$ & 7.27E-06 & $4.46 \mathrm{E}-04$ & $9.95 \mathrm{E}+01$ & $4.01 \mathrm{E}+06$ & $7.72 \mathrm{E}+04$ & $6.19 \mathrm{E}+01$ & $3.79 \mathrm{E}+03$ \\
\hline 6 & 2 & 4 & 2 & $3.48 \mathrm{E}+08$ & $6.10 \mathrm{E}-01$ & $7.02 \mathrm{E}-03$ & $3.96 \mathrm{E}-06$ & $2.75 \mathrm{E}-04$ & $9.94 \mathrm{E}+01$ & $2.12 \mathrm{E}+06$ & $2.45 \mathrm{E}+04$ & $1.38 \mathrm{E}+01$ & $9.57 \mathrm{E}+02$ \\
\hline 7 & 2 & 4 & 3 & $6.09 \mathrm{E}+08$ & $1.73 \mathrm{E}-01$ & $4.79 \mathrm{E}-03$ & $9.93 \mathrm{E}-07$ & $2.44 \mathrm{E}-04$ & $9.98 \mathrm{E}+01$ & $1.05 \mathrm{E}+06$ & $2.91 \mathrm{E}+04$ & $6.05 \mathrm{E}+00$ & $1.49 \mathrm{E}+03$ \\
\hline 8 & 2 & 4 & 4 & $4.30 \mathrm{E}+08$ & $3.90 \mathrm{E}-01$ & $6.21 \mathrm{E}-02$ & $2.18 \mathrm{E}-06$ & $3.15 \mathrm{E}-05$ & $9.95 \mathrm{E}+01$ & $1.68 \mathrm{E}+06$ & $2.67 \mathrm{E}+05$ & $9.38 \mathrm{E}+00$ & $1.35 \mathrm{E}+02$ \\
\hline 9 & 3 & 5 & 1 & $2.90 \mathrm{E}+08$ & $3.30 \mathrm{E}-01$ & $2.38 \mathrm{E}-02$ & $1.64 \mathrm{E}-06$ & $1.49 \mathrm{E}-04$ & $9.96 \mathrm{E}+01$ & $9.57 \mathrm{E}+05$ & $6.91 \mathrm{E}+04$ & $4.76 \mathrm{E}+00$ & $4.32 \mathrm{E}+02$ \\
\hline 10 & 3 & 5 & 2 & $6.68 \mathrm{E}+08$ & 4.54E-01 & $2.75 \mathrm{E}-02$ & $6.37 \mathrm{E}-06$ & $9.46 \mathrm{E}-05$ & $9.95 \mathrm{E}+01$ & $3.03 \mathrm{E}+06$ & $1.83 \mathrm{E}+05$ & $4.26 \mathrm{E}+01$ & $6.32 \mathrm{E}+02$ \\
\hline 11 & 3 & 5 & 3 & $5.03 \mathrm{E}+08$ & $3.92 \mathrm{E}-01$ & $1.37 \mathrm{E}-02$ & 4.65E-06 & $1.64 \mathrm{E}-04$ & $9.96 \mathrm{E}+01$ & $1.97 \mathrm{E}+06$ & $6.91 \mathrm{E}+04$ & $2.34 \mathrm{E}+01$ & $8.26 \mathrm{E}+02$ \\
\hline 12 & 3 & 5 & 4 & $9.85 \mathrm{E}+07$ & $6.24 \mathrm{E}-01$ & $7.29 \mathrm{E}-03$ & $1.46 \mathrm{E}-05$ & $2.11 \mathrm{E}-04$ & $9.94 \mathrm{E}+01$ & $6.15 \mathrm{E}+05$ & $7.17 \mathrm{E}+03$ & $1.44 \mathrm{E}+01$ & $2.08 \mathrm{E}+02$ \\
\hline 13 & 3 & 5 & 5 & $6.98 \mathrm{E}+07$ & $2.61 \mathrm{E}-01$ & $6.38 \mathrm{E}-03$ & $2.06 \mathrm{E}-05$ & $7.55 \mathrm{E}-05$ & $9.97 \mathrm{E}+01$ & $1.82 \mathrm{E}+05$ & $4.45 \mathrm{E}+03$ & $1.44 \mathrm{E}+01$ & $5.27 \mathrm{E}+01$ \\
\hline
\end{tabular}


$\boldsymbol{A}$

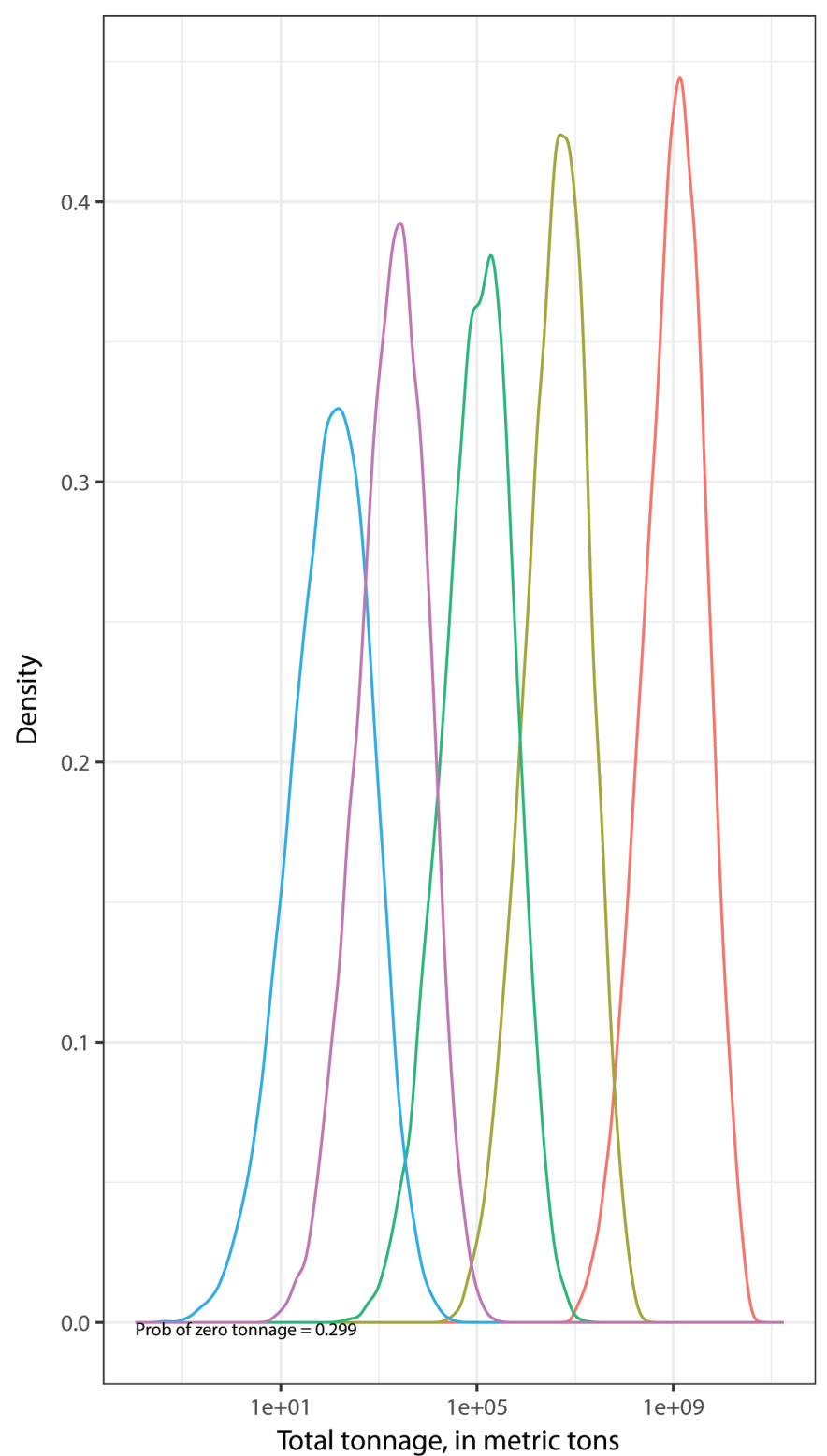

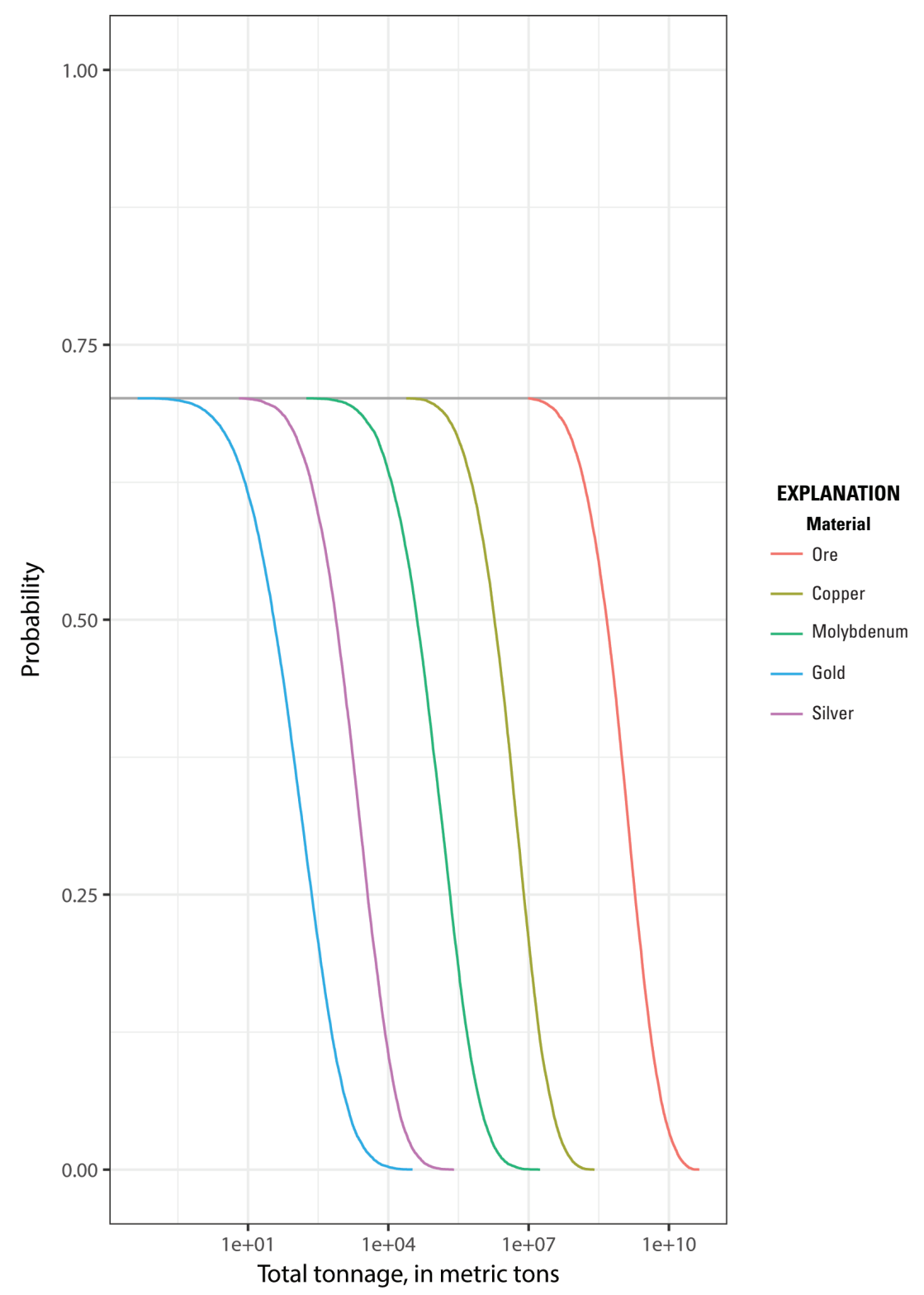

Figure 6. $A$, Univariate, marginal, probability density functions and $B$, Univariate, marginal, complementary cumulative distribution functions for the total ore and mineral resource tonnages in all undiscovered deposits within the permissive tract. 


\section{User's Guide for MapMark4GUI—A Graphical User Interface for the MapMark4 R Package}

Table 4. Simulation results aggregated by simulation run index.

[The "Ore_MetricTons" column includes the ore tonnage in metric tons, and the contained tonnages of each commodity included in the grade and tonnage model are listed in metric tons in the columns "C $\mathrm{Cu}$ _MetricTons" through "Ag_MetricTons." $\mathrm{Cu}$, copper; Mo, molybdenum; Au, gold; $\mathrm{Ag}$, silver]

\begin{tabular}{ccccccc}
\hline Simulation Index & $\begin{array}{c}\text { Number of } \\
\text { Deposits }\end{array}$ & Ore_MetricTons & Cu_MetricTons & Mo_MetricTons & Au_MetricTons & Ag_MetricTons \\
\hline 1 & 4 & $4.51 \mathrm{E}+09$ & $2.06 \mathrm{E}+07$ & $4.29 \mathrm{E}+05$ & $7.73 \mathrm{E}+02$ & $8.54 \mathrm{E}+03$ \\
2 & 4 & $2.24 \mathrm{E}+09$ & $8.87 \mathrm{E}+06$ & $3.98 \mathrm{E}+05$ & $9.11 \mathrm{E}+01$ & $6.37 \mathrm{E}+03$ \\
3 & 5 & $1.63 \mathrm{E}+09$ & $6.75 \mathrm{E}+06$ & $3.33 \mathrm{E}+05$ & $9.95 \mathrm{E}+01$ & $2.15 \mathrm{E}+03$ \\
4 & 8 & $1.16 \mathrm{E}+10$ & $5.22 \mathrm{E}+07$ & $9.31 \mathrm{E}+05$ & $9.30 \mathrm{E}+02$ & $2.92 \mathrm{E}+04$ \\
5 & 5 & $4.39 \mathrm{E}+09$ & $1.63 \mathrm{E}+07$ & $6.30 \mathrm{E}+05$ & $3.06 \mathrm{E}+02$ & $8.58 \mathrm{E}+03$ \\
\hline
\end{tabular}

Table 5. Numbers of estimated undiscovered deposits and their relative probabilities.

[The "NDeposits" column lists the numbers of estimated undiscovered deposits. The "RelProb" column gives the relative probabilities]

\begin{tabular}{|c|c|}
\hline NDeposits & RelProbs \\
\hline 0 & 0.300 \\
\hline 1 & 0.400 \\
\hline 2 & 0.225 \\
\hline 3 & 0.045 \\
\hline 4 & 0.030 \\
\hline
\end{tabular}


Probability of zero tonnage $=0.299$
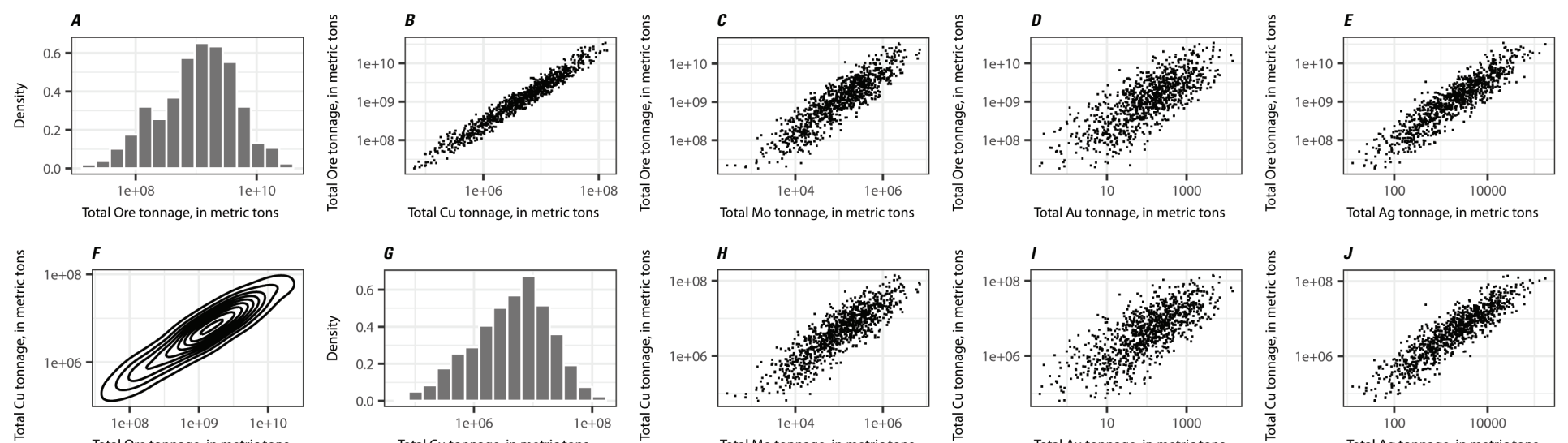

Total Mo tonnage, in metric ton

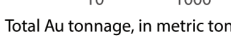

Total Ag tonnage, in metric tons
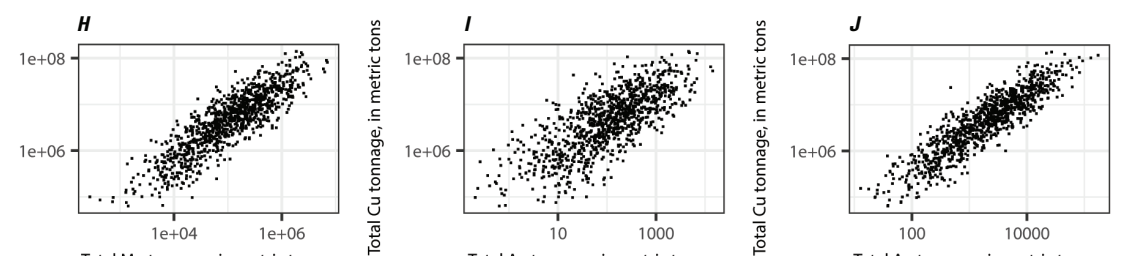

Total Cu tonnage, in metric tons
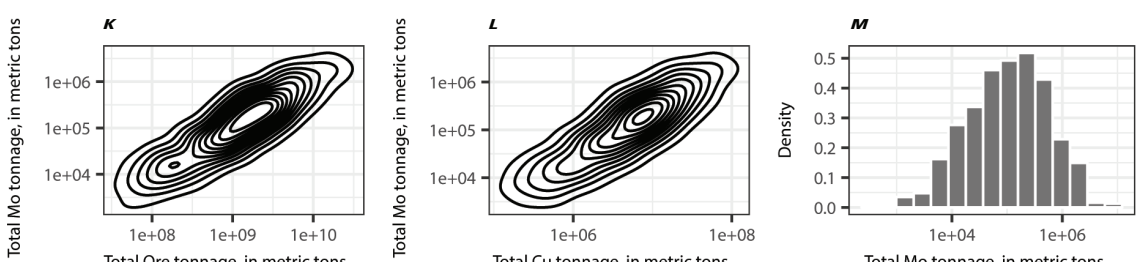

Tota

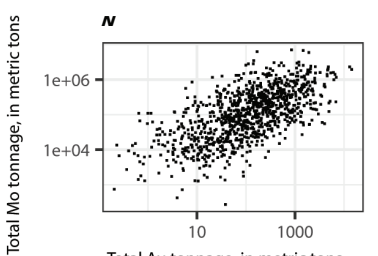

Total Cu tonnage, in metric tons

Total Mo tonnage, in metric tons
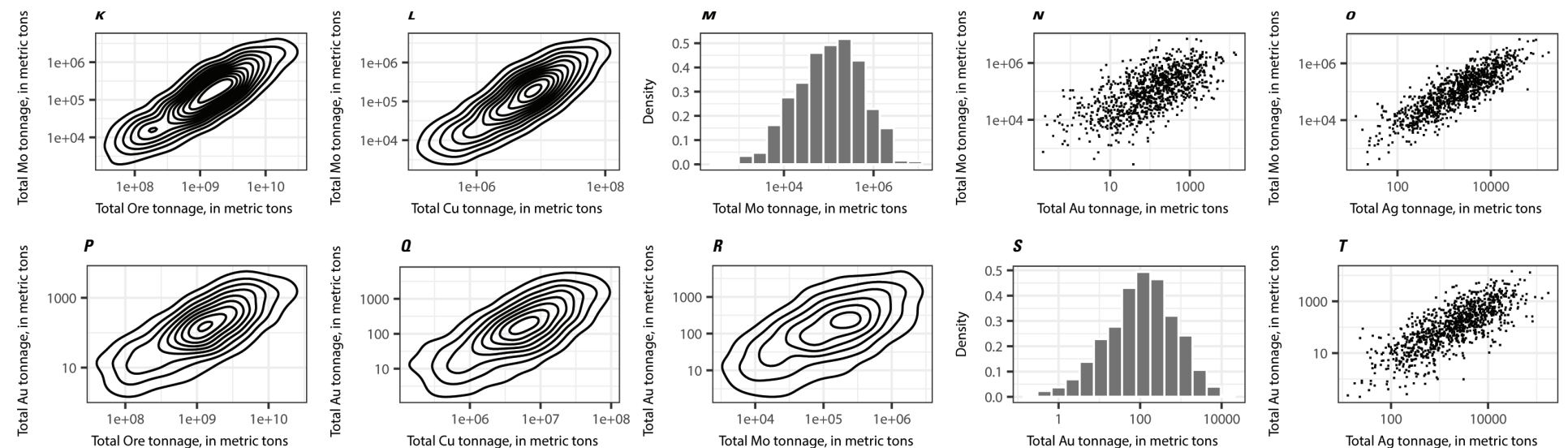

Total Ag tonnage, in metric tons
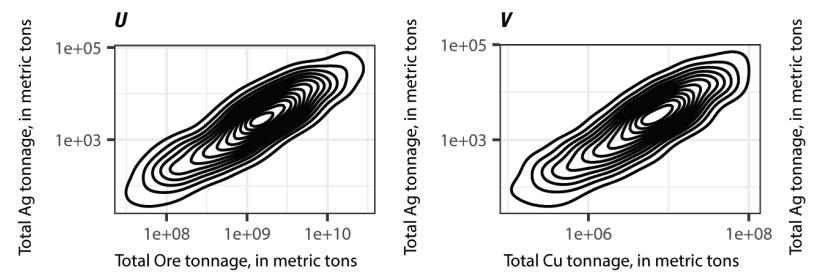

$w$

$\boldsymbol{X}$
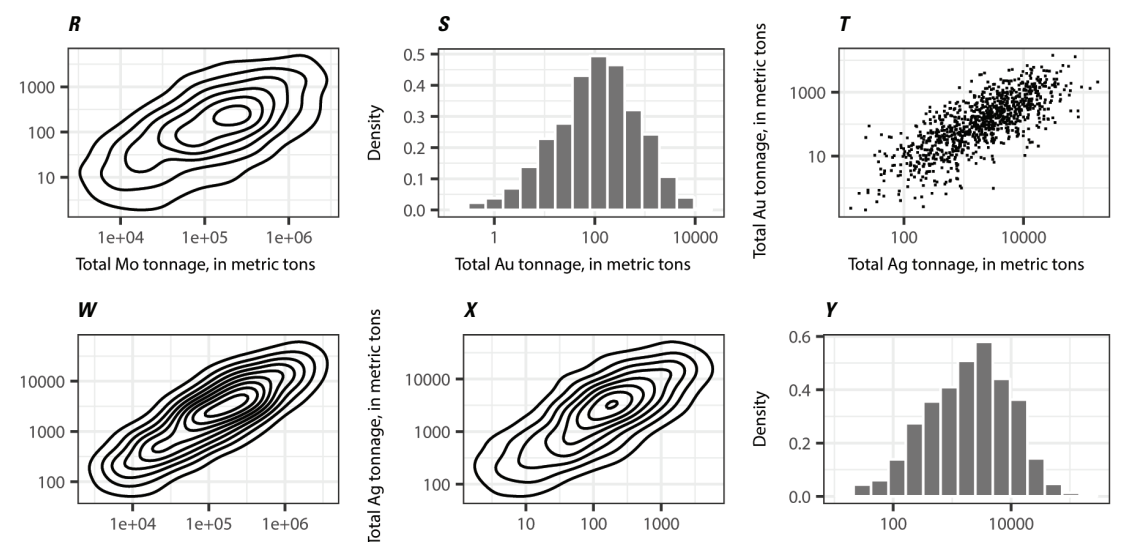

$\begin{array}{lll}2 l e+04 & 1 \mathrm{e}+05 & 1 \mathrm{e}+06\end{array}$
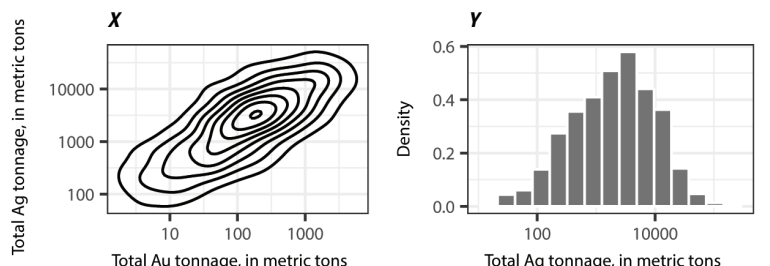

Figure 7. $A-Y$, Matrix graphic showing the univariate and bivariate marginal distributions for the ore and mineral resource tonnages in all undiscovered deposits within the permissive tract. The probability of zero tonnage is printed above the plot matrix. Three types of plots are in this matrix: (1) plots in the upper triangle show the cross-plots of the ore tonnage and commodity tonnages, (2) plots along the diagonal show the histograms representing the univariate marginal distributions, and (3) plots in the lower triangle show the bivariate marginal distributions of the simulation results (Ellefsen, 2017b). The example is for a porphyry copper grade and tonnage model that includes the commodities copper (Cu), molybdenum (Mo), gold ( $\mathrm{Au}$ ), and silver $(\mathrm{Ag})$. The source is a subset of data from the Singer and others (2008) porphyry copper model that is not missing values. 
Clicking the "Download PMF Stats" button also generates the statistics for the deposit PMF, summarizing the number of undiscovered deposits in the tract. The statistics include the mean, variance, and information entropy. Information entropy is the uncertainty measure for the PMF. The higher the uncertainty, the greater the information entropy value (McElreath, 2016). The resulting statistical output is saved as a CSV file named "RunID_04_PMF_Stats.csv" (table 6).

Clicking the "Download Sim and GTM Stats" button generates statistics for the GTM and the simulation results. The statistics for the input GTM (means, maximums, minimums, medians, standard deviations, and the percentiles of the ore tonnage and grades) are saved as a file named "RunID_02 GTM_Stats.csv" (table 7).

The simulation statistics include the ore tonnage, commodity grades, and contained in-ground commodity tonnage for the simulated undiscovered deposits. Reported statistics are similar to those reported for the GTM; however, they refer to the simulated undiscovered deposits instead of the GTM. The process uses the simulation results from the simulation output file (RunID_05_SIM_EF.csv) and writes the statistics to a file named "RuñID_06_SIM_EF_Stats.csv" (table 8).

The final statistical analysis for the ore tonnage and contained in-place resource tonnages for the estimated undiscovered resources is generated from aggregating individual deposits by simulation run index. Before the statistics can be run, a pivot table aggregation calculation using the simulation run index as the base is done on the simulated deposits results file (RunID_05_SIM_EF.csv). The simulated deposits file can be used for an economic filter analysis. The aggregation generates the contained totals file (RunID_07_SIM_Contained Totals.csv). Using the aggregated simulated deposits output, statistics are calculated for the contained resources, along with the probability of zero resources and the probability of contained resources greater than or equal to the mean. The output file of this aggregated statistical analysis is named “RunID_08_SIM_Contained_Stats.csv" (table 9).

\section{Plots}

Running the MapMark4 processes develops plots for the probability calculation and simulation results. When running each process using MapMark4GUI, the plots are shown on the $\mathrm{R}$ console screen immediately after each process run is complete. The plots are also saved in the output folder as EPS

Table 6. Statistics for the probability mass function results.

[The table includes the probability mass function statistics based on the number of undiscovered deposits. The first column includes the mean, the second column lists the variance, and the third column gives the information entropy for the probability mass function results]

\begin{tabular}{ccc}
\hline meanopmf & varopmf & InformationEntropy \\
\hline 1.105 & 0.963975 & 1.308076394 \\
\hline
\end{tabular}

and JPEG files. MapMark4GUI enables the user to review each of the plots in any order after all processes are complete. This is done by clicking the buttons in the "Plots" section of the MapMark Inputs dialog. Clicking any of the plot buttons will relaunch the plot onto the $\mathrm{R}$ console screen. The plots can be viewed repeatedly to support visual analysis.

\section{Output Files}

MapMark4GUI generates 14 files that are saved in the output folder, including input parameters, statistics, and plot graphics. The first eight files are output as CSV tables. The fifth and sixth files have "EF" in the names because they can support economic filter analysis.

- RunID_01_InputParameters.csv-The MapMark4GUI parameters that were entered for the simulation run.

- RunID_02_GTM_Stats.csv-Statistics for the input GTM that summarize the means, maximums, minimums, medians, standard deviations, and percentiles of the ore tonnage and commodity grades.

- RunID_03_PMF_Probs.csv-The numbers (N) of deposits and relative probabilities for the PMF model.

- RunID_04_PMF_Stats.csv_-Statistics for the PMF, including the mean number of deposits, variance, and information entropy.

-RunID_05_SIM_EF.csv-Simulation run results of each deposit that can be used for further data analysis, such as applying economic filters.

- RunID_06_SIM_EF_Stats.csv-Statistics for the information contained in the RunID_05_SIM_EF.csv file. This statistics file summarizes the means, maximums, minimums, medians, standard deviations, and percentiles of the simulated ore tonnages, commodity grades, and contained resources.

- RunID_07_SIM_Contained_Totals.csv-Contained ore and commodity tonnages aggregated by simulation run index.

- RunID_08_SIM_Contained_Stats.csv-Statistics for the simulation contained totals results summarizing the means, maximums, minimums, medians, standard deviations, percentiles of the ore and commodity contained resource tonnages, the probability of zero resources, and probability of resources greater than or equal to the mean.

MapMark4GUI also outputs five plot graphics in EPS and JPEG formats. These plots show the resulting probability functions and simulation plots.

- RunIDGradPdfPlot.eps and RunIDGradPdfPlot.jpg-A plot showing the PDF results for the grades. 
Table 7. Statistics for the input grade and tonnage model.

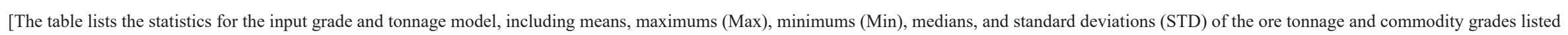

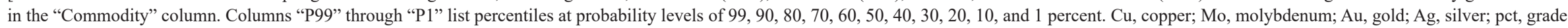
percent]

\begin{tabular}{|c|c|c|c|c|c|c|c|c|c|c|c|c|c|c|c|c|}
\hline Commodity & Means & Max & Min & Median & STD & P99 & P90 & P80 & P70 & P60 & P50 & P40 & P30 & P20 & P10 & P1 \\
\hline \multicolumn{17}{|l|}{ Ore } \\
\hline$\overline{\text { MetricTons }}$ & $1.77 \mathrm{E}+09$ & $3.08 \mathrm{E}+10$ & $9.61 \mathrm{E}+06$ & $7.22 \mathrm{E}+08$ & $3.99 \mathrm{E}+09$ & $1.53 \mathrm{E}+07$ & $8.69 \mathrm{E}+07$ & $2.00 \mathrm{E}+08$ & $2.72 \mathrm{E}+08$ & $4.09 \mathrm{E}+08$ & $7.22 \mathrm{E}+08$ & $8.77 \mathrm{E}+08$ & $1.42 \mathrm{E}+09$ & $1.99 \mathrm{E}+09$ & $3.23 \mathrm{E}+09$ & $2.12 \mathrm{E}+10$ \\
\hline $\mathrm{Cu} \_$pct & 0.44079 & 0.88200 & 0.12000 & 0.42100 & 0.15129 & 0.14560 & 0.26000 & 0.33000 & 0.36000 & 0.39000 & 0.42100 & 0.47300 & 0.50000 & 0.56000 & 0.63000 & 0.85000 \\
\hline Mo_pct & 0.01432 & 0.06300 & 0.00100 & 0.01000 & 0.01343 & 0.00100 & 0.00300 & 0.00500 & 0.00700 & 0.00800 & 0.01000 & 0.01200 & 0.01500 & 0.02000 & 0.03300 & 0.05960 \\
\hline Au_pct & 0.00002 & 0.00020 & 0.00000 & 0.00001 & 0.00002 & 0.00000 & 0.00000 & 0.00000 & 0.00000 & 0.00001 & 0.00001 & 0.00002 & 0.00002 & 0.00003 & 0.00004 & 0.00009 \\
\hline Ag pct & 0.00024 & 0.00200 & 0.00001 & 0.00017 & 0.00024 & 0.00003 & 0.00007 & 0.00010 & 0.00013 & 0.00015 & 0.00017 & 0.00020 & 0.00025 & 0.00030 & 0.00050 & 0.00098 \\
\hline
\end{tabular}

Table 8. Statistics for the Monte Carlo simulation run.

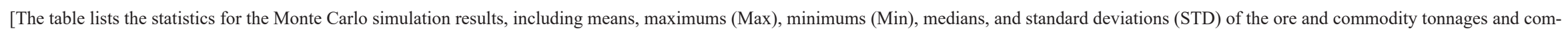

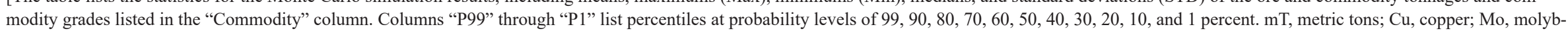
denum; Au, gold; Ag, silver; pct, grade percent]

\begin{tabular}{|c|c|c|c|c|c|c|c|c|c|c|c|c|c|c|c|c|}
\hline Commodity & Means & Max & Min & Median & STD & P99 & P90 & P80 & P70 & P60 & P50 & P40 & P30 & P20 & P10 & P1 \\
\hline \multicolumn{17}{|l|}{ Ore_ } \\
\hline MetricTons & $1.64 \mathrm{E}+09$ & $3.07 \mathrm{E}+10$ & $9.62 \mathrm{E}+06$ & $6.08 \mathrm{E}+08$ & $3.06 \mathrm{E}+09$ & $2.07 \mathrm{E}+07$ & $8.89 \mathrm{E}+07$ & $1.70 \mathrm{E}+08$ & $2.73 \mathrm{E}+08$ & $4.08 \mathrm{E}+08$ & $6.08 \mathrm{E}+08$ & $8.76 \mathrm{E}+08$ & $1.32 \mathrm{E}+09$ & $2.12 \mathrm{E}+09$ & $4.04 \mathrm{E}+09$ & $1.67 \mathrm{E}+10$ \\
\hline $\mathrm{Cu} \_$pct & 0.42991 & 0.88184 & 0.12119 & 0.40827 & 0.14894 & 0.17566 & 0.25405 & 0.29905 & 0.33700 & 0.37171 & 0.40827 & 0.44792 & 0.49415 & 0.55252 & 0.64061 & 0.82987 \\
\hline Mo_pct & 0.01326 & 0.06293 & 0.00101 & 0.00963 & 0.01139 & 0.00136 & 0.00299 & 0.00442 & 0.00592 & 0.00761 & 0.00963 & 0.01204 & 0.01531 & 0.02024 & 0.02929 & 0.05395 \\
\hline $\mathrm{Au} \_p c t$ & 0.00002 & 0.00020 & 0.00000 & 0.00001 & 0.00003 & 0.00000 & 0.00000 & 0.00000 & 0.00000 & 0.00001 & 0.00001 & 0.00001 & 0.00002 & 0.00003 & 0.00005 & 0.00015 \\
\hline Ag_pct & 0.00023 & 0.00198 & 0.00001 & 0.00017 & 0.00020 & 0.00003 & 0.00006 & 0.00009 & 0.00012 & 0.00014 & 0.00017 & 0.00021 & 0.00026 & 0.00033 & 0.00046 & 0.00099 \\
\hline gangue_pct & 99.55658 & 99.85426 & 99.07652 & 99.57893 & 0.14923 & 99.15671 & 99.34637 & 99.43311 & 99.49246 & 99.53800 & 99.57893 & 99.61483 & 99.64972 & 99.68730 & 99.73249 & 99.81263 \\
\hline \multicolumn{17}{|l|}{$\mathrm{Cu}_{-}$} \\
\hline MetricTons & $7.09 \mathrm{E}+06$ & $2.42 \mathrm{E}+08$ & $2.03 \mathrm{E}+04$ & $2.42 \mathrm{E}+06$ & $1.42 \mathrm{E}+07$ & $7.90 \mathrm{E}+04$ & $3.39 \mathrm{E}+05$ & $6.65 \mathrm{E}+05$ & $1.08 \mathrm{E}+06$ & $1.65 \mathrm{E}+06$ & $2.42 \mathrm{E}+06$ & $3.58 \mathrm{E}+06$ & $5.42 \mathrm{E}+06$ & $8.83 \mathrm{E}+06$ & $1.75 \mathrm{E}+07$ & $7.43 \mathrm{E}+07$ \\
\hline \multicolumn{17}{|l|}{$\mathrm{Mo}_{-}$} \\
\hline MetricTons & $2.14 \mathrm{E}+05$ & $1.26 \mathrm{E}+07$ & $1.49 \mathrm{E}+02$ & $5.65 \mathrm{E}+04$ & $5.47 \mathrm{E}+05$ & $1.10 \mathrm{E}+03$ & $6.25 \mathrm{E}+03$ & $1.33 \mathrm{E}+04$ & $2.32 \mathrm{E}+04$ & $3.67 \mathrm{E}+04$ & $5.65 \mathrm{E}+04$ & $8.87 \mathrm{E}+04$ & $1.40 \mathrm{E}+05$ & $2.42 \mathrm{E}+05$ & $5.02 \mathrm{E}+05$ & $2.51 \mathrm{E}+06$ \\
\hline \multicolumn{17}{|l|}{$\mathrm{Au}_{-}$} \\
\hline MetricTons & $3.12 \mathrm{E}+02$ & $4.30 \mathrm{E}+04$ & $3.02 \mathrm{E}-02$ & $5.22 \mathrm{E}+01$ & $1.11 \mathrm{E}+03$ & $5.48 \mathrm{E}-01$ & $4.11 \mathrm{E}+00$ & $9.84 \mathrm{E}+00$ & $1.85 \mathrm{E}+01$ & $3.21 \mathrm{E}+01$ & $5.22 \mathrm{E}+01$ & $9.02 \mathrm{E}+01$ & $1.51 \mathrm{E}+02$ & $2.73 \mathrm{E}+02$ & $6.54 \mathrm{E}+02$ & $4.32 \mathrm{E}+03$ \\
\hline \multicolumn{17}{|l|}{$\mathrm{Ag}_{-}$} \\
\hline MetricTons & $3.74 \mathrm{E}+03$ & $4.65 \mathrm{E}+05$ & $2.98 \mathrm{E}+00$ & $1.04 \mathrm{E}+03$ & $9.79 \mathrm{E}+03$ & $2.29 \mathrm{E}+01$ & $1.23 \mathrm{E}+02$ & $2.50 \mathrm{E}+02$ & $4.23 \mathrm{E}+02$ & $6.71 \mathrm{E}+02$ & $1.04 \mathrm{E}+03$ & $1.58 \mathrm{E}+03$ & $2.51 \mathrm{E}+03$ & $4.26 \mathrm{E}+03$ & $8.83 \mathrm{E}+03$ & $4.16 \mathrm{E}+04$ \\
\hline
\end{tabular}


[The table lists the statistics for the simulated contained resources, including means, maximums (Max), minimums (Min), medians, and standard deviations (STD) of the contained ore and commodity contained resource tonnages listed in the "Commodity" column. Columns "P99" through "P1" list percentile results at probability levels of 99, 90, 80, 70, 60, 50, 40, 30, 20, 10, and 1 percent. The "Prob of Zero" column gives the probability of zero resources, and the "Prob $\geq$ Mean" column gives the probability greater than or equal to the mean. $\geq$, greater than or equal to; Cu, copper; Mo, molybdenum; Au, gold; Ag, silver]

\begin{tabular}{lcccccccccccc}
\hline \multicolumn{1}{c}{ Commodity } & Means & Max & Min & Median & STD & P99 & P90 & P80 & P70 & P60 & P50 & P40 \\
\hline Ore_MetricTons & $1.82 \mathrm{E}+09$ & $4.11 \mathrm{E}+10$ & $0.00 \mathrm{E}+00$ & $4.72 \mathrm{E}+08$ & $3.61 \mathrm{E}+09$ & $0.00 \mathrm{E}+00$ & $0.00 \mathrm{E}+00$ & $0.00 \mathrm{E}+00$ & $1.88 \mathrm{E}+07$ & $2.07 \mathrm{E}+08$ & $4.72 \mathrm{E}+08$ & $8.58 \mathrm{E}+08$ \\
Cu_MetricTons & $7.89 \mathrm{E}+06$ & $2.56 \mathrm{E}+08$ & $0.00 \mathrm{E}+00$ & $1.86 \mathrm{E}+06$ & $1.65 \mathrm{E}+07$ & $0.00 \mathrm{E}+00$ & $0.00 \mathrm{E}+00$ & $0.00 \mathrm{E}+00$ & $6.88 \mathrm{E}+04$ & $8.17 \mathrm{E}+05$ & $1.86 \mathrm{E}+06$ & $3.46 \mathrm{E}+06$ \\
Mo_MetricTons & $2.38 \mathrm{E}+05$ & $1.27 \mathrm{E}+07$ & $0.00 \mathrm{E}+00$ & $4.09 \mathrm{E}+04$ & $6.13 \mathrm{E}+05$ & $0.00 \mathrm{E}+00$ & $0.00 \mathrm{E}+00$ & $0.00 \mathrm{E}+00$ & $9.12 \mathrm{E}+02$ & $1.65 \mathrm{E}+04$ & $4.09 \mathrm{E}+04$ & $8.29 \mathrm{E}+04$ \\
Au_MetricTons & $3.47 \mathrm{E}+02$ & $4.30 \mathrm{E}+04$ & $0.00 \mathrm{E}+00$ & $3.62 \mathrm{E}+01$ & $1.20 \mathrm{E}+03$ & $0.00 \mathrm{E}+00$ & $0.00 \mathrm{E}+00$ & $0.00 \mathrm{E}+00$ & $4.81 \mathrm{E}-01$ & $1.24 \mathrm{E}+01$ & $3.62 \mathrm{E}+01$ & $7.91 \mathrm{E}+01$ \\
Ag_MetricTons & $4.16 \mathrm{E}+03$ & $4.69 \mathrm{E}+05$ & $0.00 \mathrm{E}+00$ & $7.65 \mathrm{E}+02$ & $1.09 \mathrm{E}+04$ & $0.00 \mathrm{E}+00$ & $0.00 \mathrm{E}+00$ & $0.00 \mathrm{E}+00$ & $2.02 \mathrm{E}+01$ & $3.10 \mathrm{E}+02$ & $7.65 \mathrm{E}+02$ & $1.51 \mathrm{E}+03$ \\
\hline
\end{tabular}

\section{Table 9. Statistics for the contained resources.-Continued}

[The table lists the statistics for the simulated contained resources, including means, maximums (Max), minimums (Min), medians, and standard deviations (STD) of the contained ore and commodity contained resource tonnages listed in the "Commodity" column. Columns "P99" through "P1" list percentile results at probability levels of 99, 90, 80, 70, 60, 50, 40, 30, 20, 10, and 1 percent. The "Prob of Zero" column gives the probability of zero resources, and the "Prob $\geq$ Mean" column gives the probability greater than or equal to the mean. $\geq$, greater than or equal to; $\mathrm{Cu}$, copper; Mo, molybdenum; $\mathrm{Au}$, gold; $\mathrm{Ag}$, silver]

\begin{tabular}{lcccccc}
\hline \multicolumn{1}{c}{ Commodity } & P30 & P20 & P10 & P1 & $\begin{array}{c}\text { Prob of } \\
\text { Zero }\end{array}$ & $\begin{array}{c}\text { Prob } \geq \\
\text { Mean }\end{array}$ \\
\hline Ore_MetricTons & $1.47 \mathrm{E}+09$ & $2.57 \mathrm{E}+09$ & $5.03 \mathrm{E}+09$ & $1.94 \mathrm{E}+10$ & 0.30 & 0.26 \\
Cu_MetricTons & $6.03 \mathrm{E}+06$ & $1.08 \mathrm{E}+07$ & $2.15 \mathrm{E}+07$ & $8.55 \mathrm{E}+07$ & 0.30 & 0.25 \\
Mo_MetricTons & $1.53 \mathrm{E}+05$ & $2.87 \mathrm{E}+05$ & $6.06 \mathrm{E}+05$ & $2.85 \mathrm{E}+06$ & 0.30 & 0.23 \\
Au_MetricTons & $1.63 \mathrm{E}+02$ & $3.22 \mathrm{E}+02$ & $8.04 \mathrm{E}+02$ & $4.95 \mathrm{E}+03$ & 0.30 & 0.19 \\
Ag_MetricTons & $2.74 \mathrm{E}+03$ & $5.03 \mathrm{E}+03$ & $1.09 \mathrm{E}+04$ & $4.72 \mathrm{E}+04$ & 0.30 & 0.23 \\
\hline
\end{tabular}


- RunIDpmfPlot.eps and RunIDpmfPlot.jpg - A plot showing the PMF results representing the probability of the number of undiscovered deposits.

- RunIDSimMatrixPlot.eps and RunIDSimMatrixPlot. jpg - A matrix of plots representing the univariate and bivariate marginal distributions for the ore and commodity tonnages.

- RunIDSimTotalPlot.eps and RunIDSimTotalPlot.jpgPlots showing the density and probability of the ore and commodity tonnages.

- RunIDTonPdfPlot.eps and RunIDTonPdfPlot.jpg-A plot showing the PDF for the ore tonnage.

MapMark4GUI also outputs a text file (RunID_Summary. txt) that contains the MapMark4 package process summary results, including the input parameters and the PDF and PMF statistics.

\section{References Cited}

Bawiec, W.J., and Spanski, G.T., 2012, Quick-start guide for version 3.0 of EMINERS - Economic Mineral Resource Simulator: U.S. Geological Survey Open-File Report 2009-1057, 26 p., accessed December 27, 2017, at https://pubs.usgs.gov/of/2009/1057.

Drew, L.J., Bliss, J.D., Bowen, R.W., Bridges, N.J., Cox, D.P., DeYoung, J.H., Jr., Houghton, J.C., Ludington, S., Menzie, W.D., Page, N.J., Root, D.H., and Singer, D.A., 1986, Quantitative estimation of undiscovered mineral resources - A case study of U.S. Forest Service wilderness tracts in the Pacific mountain system: Economic Geology, v. 81 , no. 1, p. 80-88, accessed December 27, 2017, at https://doi.org/10.2113/gsecongeo.81.1.80.

Duval, J.S., 2000, A Microsoft Windows version of the MARK3 Monte Carlo resource simulator: U.S. Geological Survey Open-File Report 00-415, CD-ROM, accessed December 27, 2017, at https://pubs.usgs.gov/of/2000/ of00-415/.
Duval, J.S., 2012, Version 3.0 of EMINERS-Economic Mineral Resource Simulator: U.S. Geological Survey Open-File Report 2004-1344, accessed December 27, 2017, at https://pubs.usgs.gov/of/2004/1344. [Supersedes ver. 2.0 released 2004.]

Ellefsen, K.J., 2017a, Probability calculations for three-part mineral assessments: U.S. Geological Survey Techniques and Methods, book 7, chap. C15, 14 p., accessed December 27, 2017, at https://doi.org/10.3133/tm7C15.

Ellefsen, K.J., 2017b, User's guide for MapMark4-An $\mathrm{R}$ package for the probability calculations in three-part mineral resource assessments: U.S. Geological Survey Techniques and Methods, book 7, chap. C14, 23 p., accessed December 27, 2017, at https://doi.org/10.3133/ tm7C14.

McElreath, R., 2016, Statistical rethinking-A Bayesian course with examples in R and Stan: Boca Raton, Fla., CRC Press, 460 p.

Root, D.H., Menzie, W.D., and Scott, W.A., 1992, Computer Monte Carlo simulation in quantitative resource estimation: Nonrenewable Resources, v. 1, no. 2, p. 125-138, accessed December 27, 2017, at https://doi.org/10.1007/BF01782266.

Singer, D.A., Berger, V.I., and Moring, B.C., 2008, Porphyry copper deposits of the world-Database and grade and tonnage models, 2008: U.S. Geological Survey OpenFile Report 2008-1155, accessed December 27, 2017, at http://pubs.usgs.gov/of/2008/1155/.

Singer, D.A., and Menzie, W.D., 2010, Quantitative mineral resource assessments-An integrated approach: New York, Oxford University Press, 219 p.

Zientek, M.L., Chechetkin, V.S., Parks, H.L., Box, S.E., Briggs, D.A., Cossette, P.M., Dolgopolova, A., Hayes, T.S., Seltmann, R., Syusyura, B., Taylor, C.D., and Wintzer, N.E., 2014, Assessment of undiscovered sandstone copper deposits of the Kodar-Udokan area, Russia: U.S. Geological Survey Scientific Investigations Report 2010-5090-M, 129 p. and spatial data, accessed December 27, 2017, at https://doi.org/10.3133/sir20105090M. 



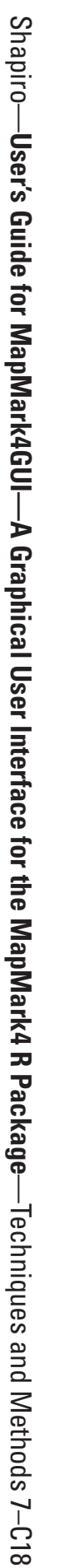

